\title{
Circumventing the Slater conundrum in countably infinite linear programs
}

\author{
Archis Ghate \\ The University of Washington, Seattle, WA 98195, USA \\ archis@uw.edu
}

March 6, 2015

\begin{abstract}
Duality results on countably infinite linear programs are scarce. Subspaces that admit an interior point, which is a sufficient condition for a zero duality gap, yield a dual where the constraints cannot be expressed using the ordinary transpose of the primal constraint matrix. Subspaces that permit a dual with this transpose do not admit an interior point. This difficulty has stumped researchers for a few decades; it has recently been called the Slater conundrum. We find a way around this hurdle.

We propose a pair of primal-dual spaces with three properties: the series in the primal and dual objective functions converge; the series defined by the rows and columns of the primal constraint matrix converge; and the order of sums in a particular iterated series of a double sequence defined by the primal constraint matrix can be interchanged so that the dual is defined by the ordinary transpose. Weak duality and complementary slackness are then immediate. Instead of using interior point conditions to establish a zero duality gap, we call upon the planning horizon method. When the series in the primal and dual constraints are continuous, we prove that strong duality holds if a sequence of optimal solutions to finite-dimensional truncations of the primal and dual CILPs has an accumulation point. We show by counterexample that the requirement that such an accumulation point exist cannot be relaxed. Our results are illustrated using several examples, and are applied to countable-state Markov decision processes and to a problem in robust optimization.
\end{abstract}

\section{Introduction}

We study infinite-dimensional linear programs (LPs) with countably infinite variables and countably infinite constraints. Following [14, 15], we call them Countably Infinite Linear Programs (CILPs). CILPs arise in infinite-horizon planning applications under time-varying technological and economic conditions; examples include production planning, equipment replacement, and capacity expansion $[22,24,37]$. They also subsume minimum cost flow problems on infinite networks [33, 38]; and LP formulations of countable-state Markov decision processes (MDPs) [15, 26, 32, 36].

We use $\mathbb{N}=\{1,2, \ldots\}$ to denote the set of all natural numbers and let $\Re^{\mathbb{N}}$ denote the space of all real-valued sequences. Variables in CILPs reside in subspaces of $\Re^{\mathbb{N}}$. Duality results for CILPs have been scarce due to numerous mathematical pathologies in $\Re^{\mathbb{N}}$. Several CILPs where weak duality fails, and where the primal and dual optimal values do not match, have been constructed [4]. An excellent recent exposition, replete with examples, of the difficulties in establishing duality results in CILPs (and more generally in infinite-dimensional LPs) is included in Martin et al. [28]. We also refer the reader to Anderson and Nash [4], Basu et al. [6], Grinold [16], and Ponstein [31] as examples of earlier works with similar discussions. We briefly review these challenges here.

(C) 2015. This manuscript version is made available under the Elsevier user license

http://www.elsevier.com/open-access/userlicense/1.0/ 

variables, the columns of the constraint matrix, the right hand side vector, and the cost coefficient vector from the primal CILP. For instance, sometimes, a natural choice for the columns of the constraint matrix and the right hand side vector is to embed them in $\Re^{\mathbb{N}}$ itself. It may then be possible to write the so-called algebraic dual problem in the space of sequences with finitely many non-zero entries by using the ordinary transpose of the primal constraint matrix. One benefit of this approach is that weak duality can be easily established when we work with the algebraic dual (see Theorem 2.1 and its single-line proof on page 18 of Anderson and Nash [4]). However, the trouble is that the non-negative orthant of $\Re^{\mathbb{N}}$ with its natural product topology has an empty interior. Thus, standard interior point conditions that ensure a zero duality gap do not hold in such CILPs (see Section 3.6.2 on page 54 of [4], and Theorem 1 on page 217 of [27]). In an attempt to remedy this situation, one may be able to embed the columns of the constraint matrix and the right hand side in a space smaller than $\Re^{\mathbb{N}}$, such as the space $l_{\infty}$ of bounded sequences, and then use the norm topology. A benefit of this approach is that the non-negative orthant in this space does have a non-empty interior. So it might be possible to close the duality gap by embedding the dual variables in the dual of $l_{\infty}$. But unfortunately, this dual space is too large - the objective function and the constraints there do not even have a series representation (see Theorem 16.31 on page 542 of [1], and Problem 2 on page 223 of [39]). Specifically, the linear operator (called the adjoint) that defines the constraints in the dual might not be the same as the ordinary transpose of the primal doubly-infinite matrix. This makes the dual structurally quite different from the familiar finite-dimensional LP dual and renders it unsatisfactory for applications. For instance, the standard finite-dimensional thought process of associating a dual variable with each primal constraint does not work, and the price-interpretation of dual variables is lost.

In short, the process of selecting appropriate subspaces for the primal and the dual CILPs is torn between two competing alternatives. We want to select sequence spaces such that the ordinary transpose of the primal constraint matrix can be seen as its adjoint and so that weak duality is immediate; but we want to do this such that some sufficient condition for a zero duality gap holds. This difficulty can be viewed as a variation of what Martin et al. [28] call the "Slater conundrum." In fact, they prove the strong and elegant result that, in a large class of Riesz spaces, the conundrum cannot be resolved. If we insist on the existence of a core point (an algebraic counterpart of the topological notion of Slater's interior point condition that is sufficient for a zero duality gap), then we must face the unsavory existence of a so-called singular dual functional that precludes writing the algebraic dual with a structure and interpretation similar to the finite-dimensional case!

\subsection{Limitations of an alternative, planning horizon approach}

An alternative method that sometimes helps in proving duality results for CILPs avoids potential complications in $\Re^{\mathbb{N}}$ by working with their finite-dimensional truncations [16, 17, 18, 20, 34, 35]. The idea is to show that finite-dimensional duality properties are preserved in the limit. Grinold [16] applied this "planning horizon" method to prove the existence of optimal dual solutions to a special class of infinite-horizon problems. Grinold [17] also used it to establish weak duality in a stationary, infinite-stage linear program; he then later extended it to convex programs [18]. Grinold and Hopkins [19] applied it to a mathematical program with an arbitrary transient phase followed by an infinite-horizon stationary phase that had a dynamic Leontief structure. Jones et al. [24] employed it to derive optimal solutions to an equipment replacement problem and its dual. The broadest applications of the planning horizon approach to CILPs have appeared in Romeijn and 

constraint contained a finite number of variables, and in a dual manner, each variable appeared in a finite number of constraints. The resulting primal constraint matrix had a lower staircase structure that was exploited in duality proofs. Romeijn et al. [35] explicitly included upper bounds on primal variables, then associated dual variables with these bound constraints, and applied the planning horizon technique to establish duality results under a transversality condition. Noting the algebraic difficulties that these upper bounds introduce in establishing transversality, Romeijn and Smith [34] proved weak duality and complementary slackness without including explicit bounds in their formulation. Strong duality was then established under transversality between accumulation points of sequences drawn from the sets of finite-dimensional primal and dual optimal solutions. Ghate and Smith [15] and Lee et al. [25] used the planning horizon method to prove duality results for non-stationary infinite-horizon Markov decision processes, heavily exploiting the structure of the constraints that appear in CILP formulations of these problems. As such, the papers that have used the planning horizon approach do not exploit the benefits (such as simpler statements of results, simpler and shorter proofs, similarities to finite-dimensional as well as to more general infinite-dimensional LPs) of a generic choice of sequence spaces. Instead, they appear to have focused either on problems that arise in specific applications or on constraint matrices with special structure to derive duality results.

\subsection{Our contributions}

In light of this literature review, it is unclear whether it is even possible to select a generic pair of sequence spaces for CILPs such that the ordinary transpose of the primal constraint matrix can be seen as its adjoint, and still ensure that some sufficient condition for a zero duality gap holds. From a modeling perspective, it is unclear whether or not any potential choice of sequence spaces will be broad enough to encompass nontrivial applications. It would thus seem that there is no way around the Slater conundrum for general CILPs.

In this paper, we attempt to put these concerns to rest. Our approach is to apply two methods to simultaneously chip away at the two sides of the conundrum. We combine the classical technique of choosing appropriate linear subspaces so that weak duality is immediate, with the planning horizon method. The former is known to work in abstract settings but has not been successfully applied previously to establish weak duality in general CILPs; the latter is known to work for establishing a zero duality gap in CILPs with a lower staircase structure. We show that, for general CILPs, the bridge between the two is a simple result in real analysis (Lemma 2.1).

Specifically, our primal and dual sequence spaces are chosen to possess three properties: (i) the series in the primal and dual objective functions converge, (ii) the series defined by each row and each column of the primal doubly-infinite constraint matrix converge, and (iii) the order of sums in a particular iterated series of a double sequence that arises in all of our proofs can be interchanged. We show that CILPs defined this way behave essentially as finite-dimensional LPs do. We therefore call the resulting dual the natural dual. Because of this choice of sequence spaces, our proofs of weak duality and complementary slackness are immediate as in the finite-dimensional case and do not need any additional conditions. Also, unlike the planning horizon approach, these proofs do not use a limiting argument. In fact, we show that it is always possible to select primal and dual sequence spaces with the above three properties; thus, our weak duality and complementary slackness results hold in every CILP.

We then switch to the planning horizon technique to prove strong duality using the natural product topologies on our sequence spaces. We prove that, when the series in the constraints in the original primal and dual CILPs are continuous, accumulation points (if they exist) of any sequence of 
optimal solutions to increasingly larger finite-dimensional truncations of the original CILPs satisfy complementary slackness conditions and are feasible. Thus, these accumulation points are optimal to the primal and dual CILPs, and they have identical objective function values; that is, strong duality holds. We show by counterexample that the requirement that such accumulation points exist cannot be relaxed. The continuity condition is trivially met in all CILPs where the constraints include a finite number of variables and variables appear in a finite number of constraints as in $[34,35]$. Thus, we recover strong duality in that paper as a special case. We also prove that the Lagrangian function of our CILPs is a linear support of the primal optimal value function. We conclude our theoretical results by deriving a saddle point property for our CILPs. To demonstrate in detail the steps involved in applying our theory, we study CILP formulations of countable-state Markov decision processes, and specifically, a stochastic equipment replacement problem within this framework. In countable-state MDPs, we are able to derive a pair of feasible primal-dual solutions using Bellman's equations of dynamic programming to concretely demonstrate strong duality. As this may not always be possible in other applications, we provide further insights into our theory by explicitly checking our sufficient conditions for strong duality in a robust auction-design problem.

\section{Problem formulation, weak duality, complementary slackness}

Let $b, c$ be in $\Re^{\mathbb{N}}$, and for $i=1,2, \ldots$, let $A_{i}$. $=\left(a_{i 1}, a_{i 2}, \ldots\right) \in \Re^{\mathbb{N}}$ be the $i$ th row of a doubly-infinite matrix $A$. Here, $A, b, c$ constitute our problem data and we do not place any restrictions on their structure. Unlike the abstract framework of Anderson and Nash [4] for general infinite-dimensional LPs, we do not need to choose subspaces for these entities; instead, our primal and dual variable spaces will be chosen such that the two resulting CILPs are "well-posed" given this problem data. Specifically, as we explain in detail next, five hypotheses labeled H1-H5 hold for our variable spaces.

Let $X \subseteq \Re^{\mathbb{N}}$ be the subset of all sequences $x \in \Re^{\mathbb{N}}$ for which

H1. the series $C(x) \triangleq \sum_{j=1}^{\infty} c_{j} x_{j}$ converges; and

H2. the series $A_{i} .(x) \triangleq \sum_{j=1}^{\infty} a_{i j} x_{j}$ converge for all $i$.

H1 was used in [34]. H2 was not needed in [34, 35] as it holds for all sequences in $\Re^{\mathbb{N}}$ if each constraint includes a finite number of variables.

Subset $X$ is in fact a linear subspace of $\Re^{\mathbb{N}}$ because it is easy to see that for any real numbers $\lambda_{1}, \lambda_{2}$, the sequence $\lambda_{1} x(1)+\lambda_{2} x(2) \in \Re^{\mathbb{N}}$ belongs to $X$ if the sequences $x(1)$ and $x(2)$ do (see Section 2.3 on page 14 of [27]). Now consider the CILP

$$
\begin{aligned}
(P) V(P) & =\inf \sum_{j=1}^{\infty} c_{j} x_{j} \\
\sum_{j=1}^{\infty} a_{i j} x_{j} & =b_{i}, \quad i=1,2, \ldots, \\
x_{j} & \geq 0, \quad j=1,2, \ldots, \\
x & \in X .
\end{aligned}
$$

We use $F \subseteq X$ to denote the (possibly empty) feasible region of $(P)$. Problems with inequality constraints and/or free variables can be converted into form $(P)$ if needed as in the finite-dimensional case, and hence $(P)$ includes all well-posed CILPs. 
We now choose a linear subspace of $\Re^{\mathbb{N}}$ to embed dual variables. The idea, as in finitedimensional LPs, is to associate a real-valued dual variable $y_{i}$ with the $i$ th constraint in $(P)$. To do this in a rigorous manner such that the resulting pair of primal-dual CILPs behaves essentially exactly like its finite-dimensional counterpart, we put some restrictions on the subspace of dual variables. Specifically, let $Y$ be the subset of all $y \in \Re^{\mathbb{N}}$ for which

H3. the series $B(y) \triangleq \sum_{i=1}^{\infty} b_{i} y_{i}$ converges;

H4. for every $x \in X$, the series $\sum_{j=1}^{\infty}\left|a_{i j} x_{j} y_{i}\right|$ converges to some limit $L_{i}\left(x, y_{i}\right)$, for $i=1,2, \ldots$; and

H5. the above limits $L_{i}\left(x, y_{i}\right)$ have the property that $\sum_{i=1}^{\infty} L_{i}\left(x, y_{i}\right)<\infty$.

H3 was used in [34]. H4 holds for all sequences $y$ in $\Re^{\mathbb{N}}$ if each constraint includes a finite number of variables as in [34, 35]. H5 need not hold for all sequences in $\Re^{\mathbb{N}}$ even when each constraint includes a finite number of variables. H5 can be seen as playing the role of the transversality conditions used in [34, 35].

Subset $Y$ is in fact a linear subspace of $\Re^{\mathbb{N}}$. To see this, suppose $\lambda_{1}, \lambda_{2}$ are two real numbers and $y(1), y(2)$ are two sequences in $Y$. We need to show that the sequence $\lambda_{1} y(1)+\lambda_{2} y(2)$ belongs to $Y$; that is, to show that H3, H4, and H5 hold for $\lambda_{1} y(1)+\lambda_{2} y(2)$. Establishing $\mathrm{H} 3$ is immediate. So we focus on H4, H5. We observe that $\sum_{j=1}^{\infty}\left|a_{i j} x_{j}\left(\lambda_{1} y_{i}(1)+\lambda_{2} y_{i}(2)\right)\right| \leq$ $\left.\left|\lambda_{1}\right| \sum_{j=1}^{\infty}\left|a_{i j} x_{j} y_{i}(1)\right|+\left|\lambda_{2}\right| \sum_{j=1}^{\infty} \mid a_{i j} x_{j} y_{i}(2)\right)|=| \lambda_{1}\left|L_{i}\left(x, y_{i}(1)\right)+\right| \lambda_{2} \mid L_{i}\left(x, y_{i}(2)\right)$. Thus, the series $\sum_{j=1}^{\infty}\left|a_{i j} x_{j}\left(\lambda_{1} y_{i}(1)+\lambda_{2} y_{i}(2)\right)\right|$ converges to some limit $L_{i}\left(x,\left(\lambda_{1} y_{i}(1)+\lambda_{2} y_{i}(2)\right)\right)$, for each $i$. Thus, H4 holds. Moreover,

$$
\sum_{i=1}^{\infty} L_{i}\left(x,\left(\lambda_{1} y_{i}(1)+\lambda_{2} y_{i}(2)\right)\right) \leq\left|\lambda_{1}\right| \sum_{i=1}^{\infty} L_{i}\left(x, y_{i}(1)\right)+\left|\lambda_{2}\right| \sum_{i=1}^{\infty} L_{i}\left(x, y_{i}(2)\right)<\infty
$$

as required for $\mathrm{H} 5$.

The following result plays a central role throughout this paper.

Lemma 2.1. Fix any $x \in X$ and any $y \in Y$. Then, (i) for each fixed $j$, the series $\sum_{i=1}^{\infty} a_{i j} y_{i} x_{j}$ converges absolutely; and (ii) the two iterated series $\sum_{i=1}^{\infty} \sum_{j=1}^{\infty} a_{i j} x_{j} y_{i}$ and $\sum_{j=1}^{\infty} \sum_{i=1}^{\infty} a_{i j} y_{i} x_{j}$ converge with

$$
\sum_{i=1}^{\infty} \sum_{j=1}^{\infty} a_{i j} x_{j} y_{i}=\sum_{j=1}^{\infty} \sum_{i=1}^{\infty} a_{i j} y_{i} x_{j}
$$

Proof. This result is a restatement of Theorem 8.43 in [5] for the double sequence $a_{i j} x_{j} y_{i}$.

Equation (5) can be interpreted as saying the desirable property that the usual transpose of the doubly-infinite matrix $A$ is its adjoint (see Section 2.2.2 in [4]). This property of $X$ and $Y$ allows us to write the dual of $(P)$ using the usual finite-dimensional thought process (see Table 4.1 on page 143 of [8]). As we shall see, it permits us to interchange the order of sums that appear 
in our proofs of weak duality and complementary slackness. This renders these proofs identical to the finite-dimensional case. The importance of this type of interchange in the order of sums (akin to Fubini's theorem) for establishing duality results is also discussed in the author's doctoral dissertation [13], in Martin et al. [28], and by Ponstein [31]. We will also see later in Section 4 that Equation (5) is the key link that enables us to replicate finite-dimensional proofs of standard properties of the Lagrangian function, primal optimal value function, and saddle points.

Without loss of generality, let subspace $X$ be such that for every $j$, there is at least one $x \in X$ for which $x_{j} \neq 0$. For $j=1,2, \ldots$, let $A \cdot j=\left(a_{1 j}, a_{2 j}, \ldots\right) \in \Re^{\mathbb{N}}$ denote the $j$ th column of $A$. The first conclusion in Lemma 2.1 then implies that the series $A_{\cdot j}(y) \triangleq \sum_{i=1}^{\infty} a_{i j} y_{i}$ converges for every $y \in Y$. We now write the dual of $(P)$ using the ordinary transpose of $A$ as

$$
\begin{gathered}
(D) V(D)=\sup \sum_{i=1}^{\infty} b_{i} y_{i} \\
\sum_{i=1}^{\infty} a_{i j} y_{i} \leq c_{j}, j=1,2, \ldots, \\
y \in Y .
\end{gathered}
$$

We call $(D)$ the natural dual of $(P)$ and use $G \subseteq Y$ to denote its (possibly empty) feasible region.

The natural dual yields a one-line proof of weak duality as in the finite-dimensional case (see Theorem 4.3 on page 146 of [8]) and as would the algebraic dual (see Theorem 2.1 on page 18 of [4]). However, the crucial difference is that the constraints in the algebraic dual need not have a concrete series representation; the constraints in our natural dual do. Finally, we comment that H1-H5 can be seen as concrete sufficient conditions that ensure that the variable and constraint spaces in our problems are appropriately "paired" as, for example, required for weak duality and complementary slackness in Section 3.3 of [4].

Theorem 2.2. Weak Duality: For any $x \in F$ and any $y \in G, \sum_{j=1}^{\infty} c_{j} x_{j} \geq \sum_{i=1}^{\infty} b_{i} y_{i}$. Hence $\infty \geq V(P) \geq V(D) \geq-\infty$ (here, the infimum over an empty set is interpreted as $+\infty$ and the supremum over an empty set is interpreted as $-\infty)$. Also, if $x \in F$ and $y \in G$ are such that $\sum_{j=1}^{\infty} c_{j} x_{j}=\sum_{i=1}^{\infty} b_{i} y_{i}$, then $x$ is optimal to $(P)$ and $y$ is optimal to $(D)$, and thus strong duality holds. Proof. We have,

$$
\sum_{i=1}^{\infty} b_{i} y_{i}=\sum_{i=1}^{\infty}\left(\sum_{j=1}^{\infty} a_{i j} x_{j}\right) y_{i}=\sum_{i=1}^{\infty} \sum_{j=1}^{\infty} a_{i j} x_{j} y_{i}=\sum_{j=1}^{\infty} \sum_{i=1}^{\infty} a_{i j} y_{i} x_{j}=\sum_{j=1}^{\infty}\left(\sum_{i=1}^{\infty} a_{i j} y_{i}\right) x_{j} \leq \sum_{j=1}^{\infty} c_{j} x_{j},
$$

where the first equality holds by (2), the third equality is simply (5), and the last inequality follows from (7) because $x_{j} \geq 0$. The other two conclusions then follow easily.

Before proceeding, we contrast this proof of weak duality with that in Romeijn and Smith [34]. The key intermediate step in their proof was their Lemma 3.2, which used a limiting argument that relied on the staircase structure of their constraint matrix and their Assumption 3.1. They explain that assumption as follows. The assumption "roughly requires that one be no worse off in the long run with respect to the infinite dimensional problem by ending the $N$ th period in any $N$ th period feasible state than by ending it in the 0-state." The key step in our proof of weak duality is Equation 
(5) in our Lemma 2.1 above, which is delivered directly by the properties of our sequence spaces $X$ and $Y$ as is more common in abstract weak duality results in infinite-dimensional LPs. We also contrast our proof of weak duality with that in Romeijn et al. [35]. In that paper, weak duality was established under the stronger (than that in [34]) assumption that off-diagonal matrices in the staircase eventually become non-negative. Specifically, this assumption implied that all feasible solutions of their dual CILP were feasible to sufficiently large truncations of that CILP; this fact was then used to show, via a limiting argument, that weak duality in the finite-dimensional truncations is inherited by the original CILPs in the limit.

We will say that the (possibly infeasible) solutions $x \in X$ and $y \in Y$ are complementary if they satisfy $x_{j}\left(c_{j}-\sum_{i=1}^{\infty} a_{i j} y_{i}\right)=0$ for each $j=1,2, \ldots$.

\section{Theorem 2.3. Complementary Slackness:}

1. Suppose $x \in F$ and $y \in G$, and suppose $x$ and $y$ are complementary. Then $x$ is optimal to $(P), y$ is optimal to $(D)$, and $V(P)=V(D)$. Thus, strong duality holds in this case.

2. Suppose $x$ is optimal to $(P), y$ is optimal to $(D)$, and $V(P)=V(D)$. Then $x$ and $y$ are complementary.

Proof. For the first claim, we have, $\sum_{j=1}^{\infty} x_{j}\left(c_{j}-\sum_{i=1}^{\infty} a_{i j} y_{i}\right)=0$ because every term inside the sum is zero. Therefore, $\sum_{j=1}^{\infty} c_{j} x_{j}=\sum_{j=1}^{\infty} x_{j} \sum_{i=1}^{\infty} a_{i j} y_{i}=\sum_{i=1}^{\infty} \sum_{j=1}^{\infty} a_{i j} x_{j} y_{i}=\sum_{i=1}^{\infty} b_{i} y_{i}$, where the interchange of the order of summation is allowed by (5). Thus, we have that $x \in F, y \in G$ and $\sum_{j=1}^{\infty} c_{j} x_{j}=\sum_{i=1}^{\infty} b_{i} y_{i}$. Therefore $x$ is optimal to $(P)$ and $y$ is optimal to $(D)$ by weak duality; and $V(P)=V(D)$.

Now for the second claim, $x$ and $y$ are given as optimal to $(P)$ and $(D)$ respectively, and since $V(P)=V(D)$, we have $\sum_{j=1}^{\infty} c_{j} x_{j}=\sum_{i=1}^{\infty} b_{i} y_{i}$. Since $x$ is feasible to $(P), b_{i}=\sum_{j=1}^{\infty} a_{i j} x_{j}$, for every $i$. Thus $\sum_{j=1}^{\infty} c_{j} x_{j}=\sum_{i=1}^{\infty} \sum_{j=1}^{\infty} a_{i j} x_{j} y_{i}=\sum_{j=1}^{\infty} x_{j} \sum_{i=1}^{\infty} a_{i j} y_{i}$ again by (5). Therefore, $\sum_{j=1}^{\infty} x_{j}\left(c_{j}-\sum_{i=1}^{\infty} a_{i j} y_{i}\right)=0$. Since $x$ is feasible to $(P), x_{j} \geq 0$ for every $j$. Moreover, since $y$ is feasible to $(D),\left(c_{j}-\sum_{i=1}^{\infty} a_{i j} y_{i}\right) \geq 0$ for every $j$. Thus, for the infinite sum to be zero, each term must be zero. That is, $\left(c_{j}-\sum_{i=1}^{\infty} a_{i j} y_{i}\right) x_{j}=0$ for every $j$.

It is important to note that subspaces $X$ and $Y$ with the aforementioned properties always exist; specifically, all requisite properties hold if both $X$ and $Y$ are chosen to equal the space of sequences with finitely many non-zeros (although this is not in general advisable from a strong duality or even a feasibility perspective). Consequently, we say (although perhaps somewhat vacuously) that given any problem data $A, b, c$, it is always possible to choose $X$ and $Y$ such that weak duality and complementary slackness Theorems 2.2 and 2.3 hold as stated.

We conclude this section by applying our results above to two examples.

Example 2.4. Our first example originally appeared in [38] and was also discussed in [15]. Consider the following primal CILP (that in fact corresponds to a minimum cost flow problem on an infinite network).

$$
\text { (P0) inf } \sum_{i=1}^{\infty}\left(1 / 2^{i}\right) x_{i, i+1}
$$


There is a single feasible solution to this CILP: $x_{i, i+1}^{*}=1$ for $i=1,2, \ldots$. We thus choose the space $X=l_{\infty}$ without loss of feasibility in this problem. Note then that the series in the objective function converges (absolutely) for every $x \in X$ and thus H1 holds. H2 holds trivially because each constraint include a finite number of variables. In order to write the natural dual of $(P 0)$, we need to choose a dual sequence space $Y$. We make this choice using the thought process described above. The series $\sum_{i=1}^{\infty} b_{i} y_{i}$ in this case reduces simply to $y_{1}$. Thus, we are assured that the dual objective function series will trivially converge and hence $\mathrm{H} 3$ will hold no matter what choice we make for $Y$. However, a further restriction must be placed on $Y$ to obtain the natural dual of $(P 0)$. We argue that $Y=l_{1}$, the sum of absolutely summable sequences, has the desired property. H4 holds because each constraint includes a finite number of variables. Moreover, for any $x \in l_{\infty}$ and any $y \in l_{1}$, we have, $\left|x_{1,2} y_{1}\right| \leq 2\left|y_{1}\right| \sup \left|x_{i, i+1}\right|<\infty$. Similarly, for $i=2,3, \ldots$, $\left|x_{i, i+1} y_{i}\right|+\left|x_{i-1, i} y_{i}\right| \leq 2\left|y_{i}\right| \sup _{i}\left|x_{i, i+1}\right|<\infty$. In addition, we have, $2 \sup _{i}\left|x_{i, i+1}\right| \sum_{i=1}^{\infty}\left|y_{i}\right|<\infty$. Thus, H5 holds. In particular, Lemma 2.1 applies and we write the natural dual of $(P 0)$ as

$$
\begin{gathered}
(D 0) \sup y_{1} \\
\begin{aligned}
y_{i}-y_{i+1} & \leq\left(1 / 2^{i}\right), i=1,2, \ldots, \\
y & \in l_{1} .
\end{aligned}
\end{gathered}
$$

Weak duality and complementary slackness Theorems 2.2 and 2.3 then hold for $(P 0)$ and $(D 0)$. Now consider the solution $y_{1}^{*}=1, y_{2}^{*}=1 / 2, y_{3}^{*}=1 / 4$, and so on; that is, $y_{i}^{*}=\left(1 / 2^{i-1}\right)$, for $i=1,2, \ldots$. It is easy to check that $y^{*}$ is feasible to $(D 0)$, and in fact, $y^{*}$ is complementary to the primal feasible solution $x^{*}$. Consequently, the first item in our complementary slackness Theorem 2.3 (or the third claim in our weak duality Theorem 2.2) implies that $x^{*}$ and $y^{*}$ are optimal to $(P 0)$ and $(D 0)$, respectively, and strong duality holds (the objective value of these solutions in the two problems is 1$)$.

Note that if we had dropped the restriction $y \in l_{1}$ from $(D 0)$, then even weak duality would have failed: solutions of the form $y_{i}=\theta$, for $i=1,2, \ldots$, would be feasible to the resulting "dual" and then weak duality would fail for any $\theta>1$. This suggests that we cannot drop H5.

Example 2.5. Our second example is from Romeijn et al. [35]. Our choice of sequence spaces for this example is somewhat more complicated than that in the example above. Consider the following primal CILP that we obtained after adding/subtracting slack/surplus variables $u, v, w$, $s$, and $t$ in the original CILP with variables $x, y$, and $z$ that appeared in [35].

$$
\begin{aligned}
(P 1) \inf \sum_{i=1}^{\infty}(1 / 2)^{i-1} z_{i} & \\
x_{1}-u_{1} & =1, \\
y_{1}+z_{1}-u_{2} & =1 \\
-2 y_{i-1}+x_{i}-u_{i+1} & =0, \quad i=2,3, \ldots, \\
-2 x_{i-1}+y_{i}+z_{i}-v_{i-1} & =0, \quad i=2,3, \ldots, \\
x_{i}+w_{i} & =2^{i-1}, \quad i=1,2, \ldots,
\end{aligned}
$$




$$
\begin{aligned}
y_{i}+s_{i} & =2^{i-1}, i=1,2, \ldots, \\
z_{i}+t_{i} & =1, i=1,2, \ldots, \\
x, y, z, u, v, w, s, t & \geq 0 .
\end{aligned}
$$

It is easy to see (and this is also mentioned in [35]) that the solution $x_{i}^{*}=y_{i}^{*}=2^{i-1}$ for $i=1,2, \ldots$; $z_{i}^{*}=u_{i}^{*}=v_{i}^{*}=w_{i}^{*}=s_{i}^{*}=0$ for $i=1,2, \ldots$; and $t_{i}^{*}=1$ for $i=1,2, \ldots$, is optimal to this problem with optimal objective value 0 . Let $X \subset \Re^{\mathbb{N}}$ be the subspace of real-valued sequences $\left(x_{1}, x_{2}, \ldots\right)$ with the property that $\left|x_{i}\right| \leq \tau(x) 2^{i-1}$ for some real number $\tau(x)$. Here, the motivation for including a scaling factor $\tau(x)$ in the bound is to ensure that the resulting subset is indeed a linear subspace. Without loss of optimality, we embed primal variables $x, y, u, v, w$, and $s$ each in the subspace $X$; and $z$ and $t$ in $l_{\infty}$. It is easy to see that the series in the objective function converges (absolutely) for this choice of sequence spaces and thus H1 holds. H2 holds trivially because each constraint includes a finite number of variables. In order to write the natural dual of $(P 1)$, we need to first embed the dual variables in appropriate subspaces. Toward this end, let $Y \subset \Re^{\mathbb{N}}$ be the subspace of real-valued sequences $\left(\alpha_{1}, \alpha_{2}, \ldots\right)$ with the property that $\sum_{i=1}^{\infty} 2^{i-1}\left|\alpha_{i}\right|<\infty$; we observe that $Y \subset l_{1}$ because this will be helpful later in our analysis. We embed dual variables $\alpha, \beta, \gamma$, and $\delta$ each in the subspace $Y$; and $\epsilon$ in $l_{1}$. Then, we have,

$$
\left|\alpha_{1}\right|+\left|\alpha_{2}\right|+\sum_{i=1}^{\infty} 2^{i-1}\left|\gamma_{i}\right|+\sum_{i=1}^{\infty} 2^{i-1}\left|\delta_{i}\right|+\sum_{i=1}^{\infty}\left|\epsilon_{i}\right|<\infty
$$

Thus, the series in the objective function of our natural dual converges (absolutely) and hence H3 holds. H4 holds trivially because each constraint includes a finite number of variables. Now, to see that H5 holds, we proceed as follows. First, we write,

$$
\begin{aligned}
& \left|x_{1} \alpha_{1}\right|+\left|-u_{1} \alpha_{1}\right|+\left|y_{1} \alpha_{2}\right|+\left|z_{1} \alpha_{2}\right|+\left|-u_{2} \alpha_{2}\right|+\sum_{i=2}^{\infty}\left(\left|-2 y_{i-1} \alpha_{i+1}\right|+\left|x_{i} \alpha_{i+1}\right|+\left|-u_{i+1} \alpha_{i+1}\right|\right)+ \\
& \sum_{i=2}^{\infty}\left(\left|-2 x_{i-1} \beta_{i-1}\right|+\left|y_{i} \beta_{i-1}\right|+\left|z_{i} \beta_{i-1}\right|+\left|-v_{i-1} \beta_{i-1}\right|\right)+\sum_{i=1}^{\infty}\left(\left|x_{i} \gamma_{i}\right|+\left|w_{i} \gamma_{i}\right|\right)+ \\
& \sum_{i=1}^{\infty}\left(\left|y_{i} \delta_{i}\right|+\left|s_{i} \delta_{i}\right|\right)+\sum_{i=1}^{\infty}\left(\left|z_{i} \epsilon_{i}\right|+\left|t_{i} \epsilon_{i}\right|\right) \\
& \leq\left|x_{1}\right|\left|\alpha_{1}\right|+\left|y_{1}\right|\left|\alpha_{2}\right|+\left|z_{1}\right|\left|\alpha_{2}\right|+\sum_{i=2}^{\infty}\left|x_{i}\right|\left|\alpha_{i+1}\right|+\sum_{i=2}^{\infty} 2\left|y_{i-1}\right|\left|\alpha_{i+1}\right|+\sum_{i=2}^{\infty} 2\left|x_{i-1}\right|\left|\beta_{i-1}\right|+ \\
& \sum_{i=2}^{\infty}\left|y_{i}\right|\left|\beta_{i-1}\right|+\sum_{i=2}^{\infty}\left|z_{i}\right|\left|\beta_{i-1}\right|+\sum_{i=1}^{\infty}\left|u_{i}\right|\left|\alpha_{i}\right|+\sum_{i=1}^{\infty}\left|v_{i}\right|\left|\beta_{i}\right|+\sum_{i=1}^{\infty}\left|x_{i}\right|\left|\gamma_{i}\right|+\sum_{i=1}^{\infty}\left|w_{i}\right|\left|\gamma_{i}\right|+ \\
& \sum_{i=1}^{\infty}\left|y_{i}\right|\left|\delta_{i}\right|+\sum_{i=1}^{\infty}\left|s_{i}\right|\left|\delta_{i}\right|+\sum_{i=1}^{\infty}\left|z_{i}\right|\left|\epsilon_{i}\right|+\sum_{i=1}^{\infty}\left|t_{i}\right|\left|\epsilon_{i}\right| .
\end{aligned}
$$

Here, the inequality is obtained by first collecting similar terms and then noting that the absolute value of a product is bounded above by the product of absolute values. The above expression is in turn bounded as

$$
\leq\left|x_{1}\right|\left|\alpha_{1}\right|+\left|y_{1}\right|\left|\alpha_{2}\right|+\left|z_{1}\right|\left|\alpha_{2}\right|+\tau(x) \sum_{i=2}^{\infty} 2^{i-1}\left|\alpha_{i+1}\right|+\tau(y) \sum_{i=2}^{\infty} 2^{i-1}\left|\alpha_{i+1}\right|+\tau(x) \sum_{i=2}^{\infty} 2^{i-1}\left|\beta_{i-1}\right|+
$$




$$
\begin{aligned}
& \tau(y) \sum_{i=2}^{\infty} 2^{i-1}\left|\beta_{i-1}\right|+\left(\sup _{i}\left|z_{i}\right|\right) \sum_{i=2}^{\infty}\left|\beta_{i-1}\right|+\tau(u) \sum_{i=1}^{\infty} 2^{i-1}\left|\alpha_{i}\right|+\tau(v) \sum_{i=1}^{\infty} 2^{i-1}\left|\beta_{i}\right|+\tau(x) \sum_{i=1}^{\infty} 2^{i-1}\left|\gamma_{i}\right|+ \\
& \tau(w) \sum_{i=1}^{\infty} 2^{i-1}\left|\gamma_{i}\right|+\tau(y) \sum_{i=1}^{\infty} 2^{i-1}\left|\delta_{i}\right|+\tau(s) \sum_{i=1}^{\infty} 2^{i-1}\left|\delta_{i}\right|+\left(\sup _{i}\left|z_{i}\right|\right) \sum_{i=1}^{\infty}\left|\epsilon_{i}\right|+\left(\sup _{i}\left|t_{i}\right|\right) \sum_{i=1}^{\infty}\left|\epsilon_{i}\right|
\end{aligned}
$$

This upper bound holds because $x, y, u, v, w, s \in X$ and $z, t \in l_{\infty}$. By multiplying some of the terms by a factor of two, this expression is in turn bounded above as

$$
\begin{aligned}
& \leq\left|x_{1}\right|\left|\alpha_{1}\right|+\left|y_{1}\right|\left|\alpha_{2}\right|+\left|z_{1}\right|\left|\alpha_{2}\right|+\tau(x) \sum_{i=2}^{\infty} 2^{i}\left|\alpha_{i+1}\right|+\tau(y) \sum_{i=2}^{\infty} 2^{i}\left|\alpha_{i+1}\right|+2 \tau(x) \sum_{i=2}^{\infty} 2^{i-2}\left|\beta_{i-1}\right|+ \\
& 2 \tau(y) \sum_{i=2}^{\infty} 2^{i-2}\left|\beta_{i-1}\right|+\left(\sup _{i}\left|z_{i}\right|\right) \sum_{i=2}^{\infty}\left|\beta_{i-1}\right|+\tau(u) \sum_{i=1}^{\infty} 2^{i-1}\left|\alpha_{i}\right|+\tau(v) \sum_{i=1}^{\infty} 2^{i-1}\left|\beta_{i}\right|+ \\
& \tau(x) \sum_{i=1}^{\infty} 2^{i-1}\left|\gamma_{i}\right|+\tau(w) \sum_{i=1}^{\infty} 2^{i-1}\left|\gamma_{i}\right|+\tau(y) \sum_{i=1}^{\infty} 2^{i-1}\left|\delta_{i}\right|+\tau(s) \sum_{i=1}^{\infty} 2^{i-1}\left|\delta_{i}\right|+\left(\sup _{i}\left|z_{i}\right|\right) \sum_{i=1}^{\infty}\left|\epsilon_{i}\right|+ \\
& \left(\sup _{i}\left|t_{i}\right|\right) \sum_{i=1}^{\infty}\left|\epsilon_{i}\right| .
\end{aligned}
$$

By adding positive terms to the first two series in this expression, we further bound it above as

$$
\begin{aligned}
& \leq\left|x_{1}\right|\left|\alpha_{1}\right|+\left|y_{1}\right|\left|\alpha_{2}\right|+\left|z_{1}\right|\left|\alpha_{2}\right|+\tau(x) \sum_{i=0}^{\infty} 2^{i}\left|\alpha_{i+1}\right|+\tau(y) \sum_{i=0}^{\infty} 2^{i}\left|\alpha_{i+1}\right|+2 \tau(x) \sum_{i=2}^{\infty} 2^{i-2}\left|\beta_{i-1}\right|+ \\
& 2 \tau(y) \sum_{i=2}^{\infty} 2^{i-2}\left|\beta_{i-1}\right|+\left(\sup _{i}\left|z_{i}\right|\right) \sum_{i=2}^{\infty}\left|\beta_{i-1}\right|+\tau(u) \sum_{i=1}^{\infty} 2^{i-1}\left|\alpha_{i}\right|+\tau(v) \sum_{i=1}^{\infty} 2^{i-1}\left|\beta_{i}\right|+ \\
& \tau(x) \sum_{i=1}^{\infty} 2^{i-1}\left|\gamma_{i}\right|+\tau(w) \sum_{i=1}^{\infty} 2^{i-1}\left|\gamma_{i}\right|+\tau(y) \sum_{i=1}^{\infty} 2^{i-1}\left|\delta_{i}\right|+\tau(s) \sum_{i=1}^{\infty} 2^{i-1}\left|\delta_{i}\right|+\left(\sup _{i}\left|z_{i}\right|\right) \sum_{i=1}^{\infty}\left|\epsilon_{i}\right|+ \\
& \left(\sup _{i}\left|t_{i}\right|\right) \sum_{i=1}^{\infty}\left|\epsilon_{i}\right|<\infty .
\end{aligned}
$$

Here, the last strict inequality follows from the definitions of subspaces $Y$ and $l_{1}$. Thus, H5 holds. Consequently, Lemma 2.1 applies and we write the natural dual as

$$
\begin{aligned}
(D 1) \sup & \alpha_{1}+\alpha_{2}+\sum_{i=1}^{\infty} 2^{i-1} \gamma_{i}+\sum_{i=1}^{\infty} 2^{i-1} \delta_{i}+\sum_{i=1}^{\infty} \epsilon_{i} \\
\alpha_{1}-2 \beta_{1}+\gamma_{1} & \leq 0 \\
\alpha_{i+1}-2 \beta_{i}+\gamma_{i} & \leq 0, i=2,3, \ldots, \\
\alpha_{2}-2 \alpha_{3}+\delta_{1} & \leq 0 \\
-2 \alpha_{i+2}+\beta_{i-1}+\delta_{i} & \leq 0, i=2,3, \ldots, \\
\alpha_{2}+\epsilon_{1} & \leq 1 \\
\beta_{i-1}+\epsilon_{i} & \leq(1 / 2)^{i-1}, i=2,3, \ldots, \\
\alpha, \beta & \geq 0 \\
\gamma, \delta, \epsilon & \leq 0
\end{aligned}
$$


In particular, our weak duality and complementary slackness Theorems 2.2, 2.3 hold. Now consider the solution $\alpha_{i}^{*}=\beta_{i}^{*}=\gamma_{i}^{*}=\delta_{i}^{*}=\epsilon_{i}^{*}=0$, for $i=1,2, \ldots$ It is easy to check that this solution is feasible to the natural dual. Since its objective function value is equal to the value of the aforementioned feasible solution to the primal, the third conclusion in our weak duality Theorem 2.2 implies that this solution is optimal to the dual and that strong duality holds.

\section{Strong duality}

We first present an example adapted from Martin et al. [28] to show that the results up to this point are not sufficient to guarantee a zero duality gap between $(P)$ and $(D)$.

Example 3.1. Consider the following pair of countably semi-infinite linear programs.

$$
\begin{aligned}
(P 2) \inf x_{1} & \\
x_{1}+x_{3}+x_{4}+\ldots & =1 \\
-x_{2}-\left(x_{3} / 3\right)-\left(x_{4} / 4\right)-\ldots & =0, \\
x_{j} & \geq 0, j=1,2, \ldots
\end{aligned}
$$

(D2) $\sup y_{1}$

$$
\begin{aligned}
y_{1} & \leq 1, \\
-y_{2} & \leq 0, \\
y_{1}-y_{2} / i & \leq 0, i=3,4, \ldots .
\end{aligned}
$$

Although these are semi-infinite programs, they can be seen as a (very) special case of CILPs. But note that the results in Romeijn and Smith [34] do not apply here as the constraints in $(P 2)$ include infinitely many variables. We choose $X=l_{1}$ as the subspace of primal variables. Here, H1 holds trivially. It is easy to see that the series in the two constraints of $(P 2)$ converge (absolutely) on $l_{1}$ and hence H2 holds. For $(D 2)$, we choose $Y=\Re^{2}$. Then H3 holds trivially. Now, for any fixed $x \in l_{1}$ and any fixed $y \in \Re^{2}$, we have, $\sum_{j=1}^{\infty}\left|x_{j} y_{1}\right| \leq\left|y_{1}\right| \sum_{j=1}^{\infty}\left|x_{j}\right|<\infty$. Thus, the series $\sum_{j=1}^{\infty}\left|x_{j} y_{1}\right|$ converges to some limit $L_{1}\left(x, y_{1}\right)$. Similarly, $\left|x_{2} y_{2}\right|+\sum_{j=3}^{\infty}\left|x_{j} y_{2}\right| / j \leq\left|x_{2} y_{2}\right|+\left|y_{2}\right| \sum_{j=3}^{\infty}\left|x_{j}\right|<\infty$. Thus, the series $\left|x_{2} y_{2}\right|+\sum_{j=3}^{\infty}\left|x_{j} y_{2}\right| / j$ converges to some limit $L_{2}\left(x, y_{2}\right)$. That is, H4 holds. Finally, $L_{1}\left(x, y_{1}\right)+L_{2}\left(x, y_{2}\right)$ is clearly finite. Thus, H5 holds and Lemma 2.1 applies. Thus, weak duality and complementary slackness hold for this example (although as we shall see now, the latter turns out to be vacuous because there are no feasible complementary solutions). Now observe that the only feasible solution of $(P 2)$ is where $x_{1}=1$ and $x_{j}=0$ for $j=2,3, \ldots$. Thus, the optimal value in $(P 2)$ is 1 . In $(D 2)$, constraints $(42)$ and (43) force $y_{1}=0$ no matter what value $y_{2}$ assumes. Thus, the optimal value in $(D 2)$ is 0 for a duality gap of 1 with $(P 2)$.

We now provide general sufficient conditions that exclude CILPs with the undesirable outcome in Example 3.1. 
For any increasing sequences of positive integers $N_{n}$ and $M_{n}$ for $n=1,2, \ldots$, we define an $N_{n}$-constraint- $M_{n}$-variable truncation of $(P)$ as

$$
\begin{aligned}
P(n) \quad V(P(n))=\inf \sum_{j=1}^{M_{n}} c_{j} x_{j} & \\
\sum_{j=1}^{M_{n}} a_{i j} x_{j} & =b_{i}, \quad i=1,2, \ldots, N_{n}, \\
x_{j} & \geq 0, j=1,2, \ldots, M_{n} .
\end{aligned}
$$

We denote the (possibly empty) feasible region of $P(n)$ by $F(n)$ and view it as a subset of $X$ by setting variables beyond $M_{n}$ to zero, that is, $x_{M_{n}+1}=x_{M_{n}+2}=\ldots=0$. Let $X^{*}(n) \subseteq F(n) \subseteq X$ denote the set of optimal solutions to $P(n)$. The dual of $P(n)$ is given by

$$
\begin{aligned}
D(n) \quad V(D(n))=\sup & \sum_{i=1}^{N_{n}} b_{i} y_{i} \\
& \sum_{i=1}^{N_{n}} a_{i j} y_{i} \leq c_{j}, j=1,2, \ldots, M_{n} .
\end{aligned}
$$

We denote its (possibly empty) feasible region by $G(n)$. We view $G(n)$ as a subset of $Y$ by setting variables beyond $N_{n}$ to zero, that is, $y_{N_{n}+1}=y_{N_{n}+2}=\ldots=0$. Let $Y^{*}(n) \subseteq G(n) \subseteq Y$ denote the set of optimal solutions to $D(n)$.

We comment on one difference between our truncations $P(n), D(n)$, and the truncations typically used in the planning horizon method. The planning horizon method was traditionally motivated by infinite-horizon decision problems. In fact, this is what imparts the constraint matrix a staircase structure where the $n$th block corresponds to (a vector of) decisions made in the $n$th period. Thus, it is common to create truncations using $n$-horizon approximations of the original infinite-horizon problem. Since we do not assume such a structure or interpretation for our primal problem, it is not possible to attach a meaning to our generic truncations $P(n)$ and $D(n)$. This is also the reason why it is not possible to make explicit how $N_{n}$ and $M_{n}$ depend on $n$.

We employ the product topology on (subspaces of) $\Re^{\mathbb{N}}$. Thus, a sequence $x(n) \in \Re^{\mathbb{N}}$ converges to $x \in \Re^{\mathbb{N}}$ if and only if each real-valued component $x_{i}(n)$ converges to the corresponding realvalued component $x_{i}$ in the usual sense in $\Re$. This topology, being a countable product of metric spaces $\Re$, is metrizable (see Theorem 3.36 on page 89 of [1]) and hence the notions of compactness and sequential compactness coincide (see Theorem 3.28 on page 86 of [1]).

Theorem 3.2. Strong Duality: Suppose there exist the aforementioned sequences $P(n)$ and $D(n)$ of finite-dimensional primal-dual problems and sets $\mathcal{C} \subseteq X$ and $\mathcal{K} \subseteq Y$ such that

C1. for each $n, X_{\mathcal{C}}(n) \triangleq\left(X^{*}(n) \cap \mathcal{C}\right) \neq \emptyset$;

C2. for each $n, Y_{\mathcal{K}}(n) \triangleq\left(Y^{*}(n) \cap \mathcal{K}\right) \neq \emptyset$;

C3. for each $i$, the constraint function $A_{i \cdot}(x)=\sum_{j=1}^{\infty} a_{i j} x_{j}$ from $X \rightarrow \Re$ is continuous over $\mathcal{C}$;

C4. for each $j$, the constraint function $A_{\cdot j}(y)=\sum_{i=1}^{\infty} a_{i j} y_{i}$ from $Y \rightarrow \Re$ is continuous over $\mathcal{K}$; and 
C5. there exists a sequence of pairs $\left(x^{*}(n), y^{*}(n)\right) \in\left(X_{\mathcal{C}}(n) \times Y_{\mathcal{K}}(n)\right)$ with a convergent (in the natural product topology on $X \times Y)$ subsequence $\left(x^{*}\left(n_{k}\right), y^{*}\left(n_{k}\right)\right)$ such that

$$
\lim _{k \rightarrow \infty}\left(x^{*}\left(n_{k}\right), y^{*}\left(n_{k}\right)\right)=(\bar{x}, \bar{y})
$$

for some $(\bar{x}, \bar{y}) \in(\mathcal{C} \times \mathcal{K})$.

Then $(P)$ and $(D)$ have optimal solutions $x^{*} \in \mathcal{C}$ and $y^{*} \in \mathcal{K}$ respectively, and $V(P)=V(D)$.

Proof. For each $i$, we have $\sum_{j=1}^{\infty} a_{i j} \bar{x}_{j}=b_{i}$. To see this, note that

$$
\begin{aligned}
\sum_{j=1}^{\infty} a_{i j} \bar{x}_{j} & =\sum_{j=1}^{\infty} a_{i j} \lim _{k \rightarrow \infty} x_{j}^{*}\left(n_{k}\right)=\lim _{k \rightarrow \infty} \sum_{j=1}^{\infty} a_{i j} x_{j}^{*}\left(n_{k}\right)=\lim _{k \rightarrow \infty}(\sum_{j=1}^{M_{n_{k}}} a_{i j} x_{j}^{*}\left(n_{k}\right)+\sum_{j=1+M_{n_{k}}}^{\infty} a_{i j} \underbrace{x_{j}^{*}\left(n_{k}\right)}_{0}) \\
& =\lim _{k \rightarrow \infty} \sum_{j=1}^{M_{n_{k}}} a_{i j} x_{j}^{*}\left(n_{k}\right)=b_{i} .
\end{aligned}
$$

The interchange of limit and infinite sum above is allowed by continuity of $A_{i \cdot}(x)$ over $\mathcal{C}$. The last equality follows from (45) because $\sum_{j=1}^{M_{n_{k}}} a_{i j} x_{j}^{*}\left(n_{k}\right)=b_{i}$ for all sufficiently large $k$ such that $N_{n_{k}} \geq i$.

For each $j$, we have $\sum_{i=1}^{\infty} a_{i j} \bar{y}_{i} \leq c_{j}$. To see this, note that

$$
\begin{aligned}
\sum_{i=1}^{\infty} a_{i j} \bar{y}_{i} & =\sum_{i=1}^{\infty} a_{i j} \lim _{k \rightarrow \infty} y_{i}^{*}\left(n_{k}\right)=\lim _{k \rightarrow \infty} \sum_{i=1}^{\infty} a_{i j} y_{i}^{*}\left(n_{k}\right)=\lim _{k \rightarrow \infty}(\sum_{i=1}^{N_{n_{k}}} a_{i j} y_{i}^{*}\left(n_{k}\right)+\sum_{i=1+N_{n_{k}}}^{\infty} a_{i j} \underbrace{y_{i}^{*}\left(n_{k}\right)}_{0}) \\
& =\lim _{k \rightarrow \infty} \sum_{i=1}^{N_{n_{k}}} a_{i j} y_{i}^{*}\left(n_{k}\right) \leq c_{j} .
\end{aligned}
$$

The interchange of limit and infinite sum above is allowed by continuity of $A_{\cdot j}(y)$ over $\mathcal{K}$. The last inequality follows from (48) because $\sum_{i=1}^{N_{n_{k}}} a_{i j} y_{i}^{*}\left(n_{k}\right) \leq c_{j}$ for all sufficiently large $k$ such that $M_{n_{k}} \geq j$. Thus, $\bar{x}$ is feasible to $(P)$ and $\bar{y}$ is feasible to $(D)$.

We now show that the pair $(\bar{x}, \bar{y})$ satisfies complementary slackness, that is, that $\bar{x}_{j}\left(c_{j}-\right.$ $\left.\left(\sum_{i=1}^{\infty} a_{i j} \bar{y}_{i}\right)\right)=0$ for each $j$. Observe that

$$
\begin{aligned}
\bar{x}_{j}\left(c_{j}-\sum_{i=1}^{\infty} a_{i j} \bar{y}_{i}\right) & =\left[\lim _{k \rightarrow \infty} x_{j}^{*}\left(n_{k}\right)\right]\left(c_{j}-\sum_{i=1}^{\infty} a_{i j} \lim _{k \rightarrow \infty} y_{i}^{*}\left(n_{k}\right)\right) \\
& =\left[\lim _{k \rightarrow \infty} x_{j}^{*}\left(n_{k}\right)\right]\left[\lim _{k \rightarrow \infty}(c_{j}-\sum_{i=1}^{N_{n_{k}}} a_{i j} y_{i}^{*}\left(n_{k}\right)-\sum_{i=1+N_{n_{k}}}^{\infty} a_{i j} \underbrace{y_{i}^{*}\left(n_{k}\right)}_{0})\right] \\
& =\lim _{k \rightarrow \infty} \underbrace{\left[x_{j}^{*}\left(n_{k}\right)\left(c_{j}-\sum_{i=1}^{N_{n_{k}}} a_{i j} y_{i}^{*}\left(n_{k}\right)\right)\right]}_{0 \text { for large } k}=0 .
\end{aligned}
$$



to problems $P\left(n_{k}\right)$ and $D\left(n_{k}\right)$ for $k$ large enough such that $M_{n_{k}} \geq j$. The result follows from the first conclusion in Theorem 2.3.

Conditions C1, C2, and C5 are similar to the hypotheses in Theorem 3.8 and Corollary 3.9 in Romeijn and Smith [34], and in Lemma 4.1 in Romeijn et al. [35]. Conditions C3 and C4 do not appear in those papers because they trivially hold there as each constraint included a finite number of variables and each variable appeared in a finite number of constraints. A typical way to establish C3 and C4 would be to show that $A_{i \cdot}(x)$ and $A_{\cdot j}(y)$ are uniformly convergent on $\mathcal{C}$ and $\mathcal{K}$, respectively. As we will demonstrate in Section 6 , this could be done using, for example, the Weierstrass test [5]. Also observe that $\mathrm{C} 5$ holds if both $\mathcal{C}$ and $\mathcal{K}$ are compact. By the Tychonoff product theorem (see Theorem 2.61 on page 52 of [1]), as in Corollary 3.9 in [34], $\mathcal{C}$ is compact if it is given by $\prod_{j=1}^{\infty}\left[0, u_{j}\right]$ for some sequence $u \geq 0$. Similarly, $\mathcal{K}$ is compact if it is given by $\prod_{i=1}^{\infty}\left[-v_{i}, v_{i}\right]$ for some sequence $v \geq 0$. The central idea in our proof above is identical to that of Lemma 4.1 in [35] and of Theorem 3.8 in [34]. We had to be more careful in working out the details and needed the additional conditions $\mathrm{C} 3$ and $\mathrm{C} 4$ because unlike those papers, we did not assume that each constraint includes a finite number of variables and each variable appears in a finite number of constraints.

In light of this strong duality theorem, we close this section by providing insight into why there is a duality gap between problems $(P 2)$ and $(D 2)$ presented in Example 3.1 above. A natural choice for the truncations of $(P 2)$ is to construct them using the first $n$ variables, for $n=1,2, \ldots$ This yields

$$
\begin{aligned}
(P 2(n)) \inf x_{1} & \\
x_{1}+x_{3}+x_{4}+\ldots+x_{n} & =1 \\
-x_{2}-\left(x_{3} / 3\right)-\left(x_{4} / 4\right)-\ldots-x_{n} / n & =0 \\
x_{j} & \geq 0, j=1,2, \ldots, n
\end{aligned}
$$

and its dual

$$
\begin{aligned}
(D 2(n)) \sup y_{1} & \\
y_{1} & \leq 1 \\
-y_{2} & \leq 0 \\
y_{1}-y_{2} / i & \leq 0, i=3,4, \ldots, n .
\end{aligned}
$$

The solution $x^{*}(n)=(1,0,0, \ldots) \in l_{1}$ is optimal to $(P 2(n))$ for all $n$; the solution $y^{*}(n)=(1, n) \in$ $\Re^{2}$ is optimal to $(D 2(n))$ for all $n$. It is easy to see that conditions C1-C4 in Theorem 3.2 hold if we choose $\mathcal{C}=\{(1,0,0, \ldots)\}$ (a singleton) and $\mathcal{K}=\Re^{2}$. Specifically, C1 holds because $(P 2(n))$ has an optimal solution in $\mathcal{C}$ for every $n$; similarly, $\mathrm{C} 2$ holds because $(D 2(n))$ has an optimal solution in $\mathcal{K}$ for every $n$. C3 holds trivially because $\mathcal{C}$ is a singleton. Finally, $\mathrm{C} 4$ holds because each constraint in (D2) only includes a finite number of variables. However, the sequence $y^{*}(n)=(1, n)$ in $\Re^{2}$ has no convergent subsequence (and hence of course has no subsequence that converges to an optimal solution of (D2)). Specifically, condition C5 does not hold. This discussion shows that we cannot drop C5 and still hope for a zero duality gap.

This failure of C5 to hold is not surprising. In fact, Martin et al. [28] have shown that the duality gap between $(P 2)$ and its dual cannot be closed if the dual is written using the ordinary 
transpose of the constraint matrix in $(P 2)$ (as one might naturally prefer to do to replicate finitedimensional intuition). It appears that the only way to close the duality gap between $(P 2)$ and its dual is to work in an algebraic dual space whose complete characterization is not known, where optimal dual variables cannot be interpreted as prices, and where the constraints are expressed using dual functionals that are not countably additive. Martin et al. [28] call this structure of the algebraic dual problem that would close the duality gap "undesirable."

Our results here provide structure that is sufficient for CILPs to behave similar to finitedimensional LPs. We further strengthen this statement in the next section by extending finitedimensional properties of the Lagrangian function, primal optimal value function, and saddle points.

\section{Lagrangian function, optimal value function, and saddle points}

In finite-dimensional LPs, one can define the so-called Lagrangian function and show that a primaldual pair of solutions is optimal if and only if this pair is a saddle point of the Lagrangian function. One can also define a primal optimal value function (this is a function of the right had side vector) and show that it is convex. Finally, the Lagrangian function is a linear support of the optimal value function - this last result allows a price interpretation of optimal dual variables. See [8, 10, 12, 27, 29] for detailed, insightful discussions of these elegant properties of finite-dimensional LPs. Romeijn and Smith [34] provided a transversality condition and their aforementioned Assumption 3.1 under which these properties extend to CILPs where constraints included a finite number of variables and variables appeared in a finite number of constraints. Thus, the question arises as to whether or not these properties extend to our more general CILPs. We briefly answer this question in the affirmative in this section.

Let subspaces $X$ and $Y$ be defined as in Section 2. Let $Z$ be the subspace of all $z \in \Re^{\mathbb{N}}$ such that the series $\sum_{i=1}^{\infty} z_{i} y_{i}$ converges. Note that $b \in Z$. We define the Lagrangian function as

$$
\Lambda(x, y ; z)=\sum_{j=1}^{\infty} c_{j} x_{j}+\sum_{i=1}^{\infty} y_{i}\left(z_{i}-A_{i} .(x)\right), \forall x \in X, \forall y \in Y, \forall z \in Z .
$$

For any $z \in Z$, we define $F_{z} \triangleq\left\{x \in X: A_{i} .(x)=z_{i}, i=1,2, \ldots ; x \geq 0\right\}$ as the set of feasible solutions to $(P)$ when the right hand side is $z$. Let $\phi$ be the primal optimal value function given by $\phi(z)=\inf _{x \in F_{z}} C(x)$, for $z \in Z$. Recall here that $C(x)$ was defined in the statement of H1 in Section 2 as the series in the objective function of $(P)$. One can show, by standard arguments in convex optimization, that $\phi$ is convex (see Proposition 1 in Chapter 8 on page 216 of [27]). This statement provides an extension of Lemma 4.1 in [34] to our setting. We then have the following extension of Theorem 4.2 in [34].

Theorem 4.1. Lagrangian support of the optimal value function: Suppose $x^{*}, y^{*}$ is a pair of complementary solutions feasible to $(P)$ and $(D)$, respectively. Then, $\Lambda\left(x^{*}, y^{*} ; z\right) \leq \phi(z)$ for all $z \in Z$, and $\Lambda\left(x^{*}, y^{*} ; b\right)=\phi(b)$.

Proof. Replicates standard steps as in the finite-dimensional case. Specifically, we fix any $z \in Z$ and first show that $\Lambda\left(x^{*}, y^{*} ; z\right) \leq C(x)$ for all $x \in F_{z}$. We have,

$$
\left.\Lambda\left(x^{*}, y^{*} ; z\right)=\sum_{j=1}^{\infty} c_{j} x_{j}^{*}+\sum_{i=1}^{\infty} y_{i}^{*}\left(z_{i}-A_{i} \cdot\left(x^{*}\right)\right)=\sum_{j=1}^{\infty} c_{j} x_{j}^{*}+\sum_{i=1}^{\infty} y_{i}^{*}\left(z_{i}-b_{i}\right) \quad \text { (by feasibility of } x^{*}\right)
$$




$$
\begin{aligned}
& =\sum_{i=1}^{\infty} z_{i} y_{i}^{*} \quad \text { by complementary slackness) } \\
& \left.=\sum_{i=1}^{\infty} A_{i} \cdot(x) y_{i}^{*} \quad \text { (because } x \in F_{z}\right) \\
& \left.=\sum_{i=1}^{\infty} \sum_{j=1}^{\infty} a_{i j} x_{j} y_{i}^{*}=\sum_{j=1}^{\infty} \sum_{i=1}^{\infty} a_{i j} y_{i}^{*} x_{j} \quad \text { by }(5)\right) \\
& \leq \sum_{j=1}^{\infty} c_{j} x_{j} \quad\left(\text { by feasibility of } y^{*}, \text { and because } x \geq 0\right) \\
& =C(x) .
\end{aligned}
$$

This implies that $\Lambda\left(x^{*}, y^{*} ; z\right) \leq \inf _{x \in F_{z}} C(x)=\phi(z)$ as required for the first claim. Finally, $\Lambda\left(x^{*}, y^{*} ; b\right)=$ $\sum_{j=1}^{\infty} c_{j} x_{j}^{*}+\sum_{i=1}^{\infty} y_{i}^{*}\left(b_{i}-A_{i} \cdot\left(x^{*}\right)\right)=\sum_{j=1}^{\infty} c_{j} x_{j}^{*}=\phi(b)$ as required for the second claim.

The pair $0 \leq x^{*} \in X$ and $y^{*} \in Y$ is said to be a saddle point of the Lagrangian function for $(P)$ and $(D)$ if

$$
\Lambda\left(x^{*}, y ; b\right) \leq \Lambda\left(x^{*}, y^{*} ; b\right) \leq \Lambda\left(x, y^{*} ; b\right), \forall 0 \leq x \in X, \forall y \in Y .
$$

We then have the following extension of Theorem 4.3 in [34].

Theorem 4.2. Saddle point property: The following two statements are equivalent.

1. Solutions $0 \leq x^{*} \in X$ and $y^{*} \in Y$ are a saddle point of the Lagrangian.

2. Solutions $x^{*} \in X$ and $y^{*} \in Y$ are feasible to $(P)$ and $(D)$, respectively, and complementary.

Proof. The first half of the proof replicates standard steps as in the finite-dimensional case. To show that the first statement implies the second, we proceed as follows. Suppose the first statement holds. From the definition of the Lagrangian function in (49), the first inequality in (50) implies that

$$
\sum_{i=1}^{\infty} y_{i}\left(b_{i}-A_{i} \cdot\left(x^{*}\right)\right) \leq \sum_{i=1}^{\infty} y_{i}^{*}\left(b_{i}-A_{i} .\left(x^{*}\right)\right), \forall y \in Y .
$$

This means that $A_{i} \cdot\left(x^{*}\right)=b_{i}$ for all $i$. Suppose not. Then there is an $i$, let us call it $i^{*}$, such that $A_{i^{*}} .\left(x^{*}\right) \neq b_{i^{*}}$. We consider three cases and in all three cases we define $y_{i}$ such that $y_{i}=y_{i}^{*}$ for all $i \neq i^{*}$; in all three cases, we set $y_{i^{*}}$ so as to get a contradiction to inequality (51). The first case is where $y_{i^{*}}^{*}\left(b_{i^{*}}-A_{i^{*} .}\left(x^{*}\right)\right)>0$; here, we set $y_{i^{*}}=2 y_{i}^{*}$. The second case is where $y_{i^{*}}^{*}\left(b_{i^{*}}-A_{i^{*} .}\left(x^{*}\right)\right)<0$; here, we set $y_{i^{*}}=0$. The third case is where $y_{i^{*}}^{*}=0$; here we set $y_{i^{*}}=-1$ or $y_{i^{*}}=1$, respectively, depending on whether $b_{i^{*}}-A_{i^{*}}\left(x^{*}\right)<0$ or $b_{i^{*}}-A_{i^{*}}\left(x^{*}\right)>0$. This discussion shows that $x^{*}$ is feasible to $(P)$. From the definition of the Lagrangian function in (49), the second inequality in (50) implies that

$$
\sum_{j=1}^{\infty} c_{j} x_{j}^{*}-\sum_{i=1}^{\infty} y_{i}^{*} A_{i \cdot} \cdot\left(x^{*}\right) \leq \sum_{j=1}^{\infty} c_{j} x_{j}-\sum_{i=1}^{\infty} y_{i}^{*} A_{i} \cdot(x), \quad \forall 0 \leq x \in X .
$$

That is,

$$
0 \leq \sum_{j=1}^{\infty} c_{j}\left(x_{j}-x_{j}^{*}\right)-\sum_{i=1}^{\infty} y_{i}^{*}\left(A_{i \cdot}(x)-A_{i \cdot}\left(x^{*}\right)\right), \forall 0 \leq x \in X
$$




$$
=\sum_{j=1}^{\infty}\left(c_{j}-\sum_{i=1}^{\infty} a_{i j} y_{i}^{*}\right)\left(x_{j}-x_{j}^{*}\right) \forall 0 \leq x \in X \text { (by (5)). }
$$

Fix any $j$. Define a $0 \leq x \in X$ such that $x_{k}=x_{k}^{*}$ for $k \neq j$ and $x_{j}=x_{j}^{*}+1$. Substituting this $x$ in (54), we see that $c_{j}-\sum_{i=1}^{\infty} a_{i j} y_{i}^{*} \geq 0$. This shows that $y^{*}$ is feasible to $(D)$. It remains to show that $x^{*}$ and $y^{*}$ are complementary. Toward this end, again fix any $j$. Define an $0 \leq x \in X$ such that $x_{k}=x_{k}^{*}$ for $k \neq j$ and $x_{j}=0$. Inequality (54) then implies that $\left(c_{j}-\sum_{i=1}^{\infty} a_{i j} y_{i}^{*}\right) x_{j}^{*} \leq 0$. Now define another $x$ such that $x_{k}=x_{k}^{*}$ for $k \neq j$ and $x_{j}=2 x_{j}^{*}$. Inequality (54) then implies that $\left(c_{j}-\sum_{i=1}^{\infty} a_{i j} y_{i}^{*}\right) x_{j}^{*} \geq 0$. This discussion shows that $\left(c_{j}-\sum_{i=1}^{\infty} a_{i j} y_{i}^{*}\right) x_{j}^{*}=0$. Thus, $x^{*}$ and $y^{*}$ are indeed complementary. That is, the second statement holds.

The second half of the proof replicates standard steps as in the finite-dimensional case. To show that the second statement implies the first, we proceed as follows. Suppose that the second statement holds. We first observe that

$$
\Lambda\left(x^{*}, y^{*} ; b\right)=\sum_{j=1}^{\infty} c_{j} x_{j}^{*}+\sum_{i=1}^{\infty} y_{i}^{*}\left(b_{i}-A_{i} \cdot\left(x^{*}\right)\right)=\sum_{j=1}^{\infty} c_{j} x_{j}^{*}=\sum_{i=1}^{\infty} y_{i}^{*} b_{i} .
$$

Here, the first equality follows from the definition of the Lagrangian function, the second equality follows because $b_{i}=A_{i} \cdot\left(x^{*}\right)$ by feasibility of $x^{*}$ to $(P)$ and the third equality follows by strong duality as in the complementary slackness Theorem 2.3. Now consider any fixed $0 \leq x \in X$. We have,

$$
\begin{aligned}
\Lambda\left(x, y^{*} ; b\right) & =\sum_{j=1}^{\infty} c_{j} x_{j}+\sum_{i=1}^{\infty} y_{i}^{*}\left(b_{i}-A_{i} .(x)\right)=\sum_{j=1}^{\infty} c_{j} x_{j}+\sum_{i=1}^{\infty} y_{i}^{*} b_{i}-\sum_{i=1}^{\infty} y_{i}^{*} \sum_{j=1}^{\infty} a_{i j} x_{j} \\
& =\sum_{j=1}^{\infty} c_{j} x_{j}+\sum_{i=1}^{\infty} y_{i}^{*} b_{i}-\sum_{j=1}^{\infty} \sum_{i=1}^{\infty} a_{i j} y_{i}^{*} x_{j} \quad(\text { by }(5)) \\
& \left.\geq \sum_{j=1}^{\infty} c_{j} x_{j}+\sum_{i=1}^{\infty} y_{i}^{*} b_{i}-\sum_{j=1}^{\infty} c_{j} x_{j} \quad \text { (by feasibility of } y^{*}, \text { and because } x \geq 0\right) \\
& =\sum_{i=1}^{\infty} y_{i}^{*} b_{i}=\Lambda\left(x^{*}, y^{*} ; b\right) .
\end{aligned}
$$

Now consider any fixed $y \in Y$. We have,

$$
\begin{aligned}
\Lambda\left(x^{*}, y ; b\right) & \left.=\sum_{j=1}^{\infty} c_{j} x_{j}^{*}+\sum_{i=1}^{\infty} y_{i}\left(b_{i}-A_{i} \cdot\left(x^{*}\right)\right)=\sum_{j=1}^{\infty} c_{j} x_{j}^{*} \quad \text { (by feasibility of } x^{*}\right) \\
& =\Lambda\left(x^{*}, y^{*} ; b\right) .
\end{aligned}
$$

This shows that the pair $x^{*}, y^{*}$ is a saddle point. That is, the first statement holds.

\section{Application to countable-state MDPs}

A countable-state MDP $[32,36]$ is a dynamic system that is observed by a decision maker at the beginning of each time-period to be in some state $s \in \mathcal{S}$, where $\mathcal{S} \triangleq\{0,1,2, \ldots\}$. The decision 
maker then chooses an action $a$ from a finite set $\mathcal{A}$ with cardinality $|\mathcal{A}|$. When action $a$ is chosen in state $s$, the system makes a transition to state $s^{\prime} \in \mathcal{S}$ with probability $p\left(s^{\prime} \mid s, a\right)$, incurring cost $0 \leq c\left(s, a ; s^{\prime}\right) \leq c<\infty$. Let $c(s, a)$ be the expected cost incurred on choosing action $a$ in state $s$. That is, $c(s, a)=\sum_{s^{\prime}} p\left(s^{\prime} \mid s, a\right) c\left(s, a ; s^{\prime}\right)$, and note that $0 \leq c(s, a) \leq c$. This procedure continues ad infinitum. The decision maker's goal is to find a decision rule that minimizes the total infinite-horizon discounted expected cost when the discount factor is $0<\alpha<1$. Examples that fit this framework include stochastic decision problems in equipment replacement, quality control, inventory management, investment management, queuing, and numerous others.

In this countable-state MDP, we concede that the assumption that costs are bounded by $c$, although not uncommon (see, for instance, [11, 32, 36]), could be restrictive from a practical viewpoint. This assumption can be relaxed by following a more technically demanding approach as in Assumptions 6.10.1 and 6.10.2 in [32]. For expository simplicity, we limit our discussion below, as in Chapter 2 of [36], to the easier case where costs are bounded and later conclude this section with an example adapted from [32] where this assumption holds. We believe that the simpler case here provides a sufficient demonstration of the ideas above.

\subsection{CILP formulations and a choice of sequence spaces}

Let $\beta(s)$ be a sequence of positive numbers indexed by states $s \in \mathcal{S}$ such that $\sum_{s \in \mathcal{S}} \beta(s)=1$. Let $v(s)$ be the minimum infinite-horizon discounted expected cost incurred when starting in state $s$; $v(\cdot): \mathcal{S} \rightarrow \Re$ is called the optimal cost-to-go function. It is shown in [36] that the the optimal costs-to-go equal the (unique) optimal values of variables $y(s)$ in the CILP

$$
\begin{gathered}
(D 3) \sup \sum_{s \in \mathcal{S}} \beta(s) y(s) \\
y(s)-\alpha \sum_{s^{\prime} \in \mathcal{S}} p\left(s^{\prime} \mid s, a\right) y\left(s^{\prime}\right) \leq c(s, a), \text { for } s \in \mathcal{S}, a \in \mathcal{A}, \\
y \in l_{\infty} .
\end{gathered}
$$

In particular, variables $y(s)$ satisfy $0 \leq y(s) \leq c /(1-\alpha)$ for all $s \in \mathcal{S}$ without loss of optimality. We have chosen $l_{\infty}$, the space of bounded sequences, as the variable space $Y$ in this problem. For any $y \in l_{\infty}$, the objective function series in (D3) converges (absolutely) because

$$
\sum_{s \in \mathcal{S}}|\beta(s) y(s)| \leq \sum_{s \in \mathcal{S}}|\beta(s)||y(s)| \leq\left(\sup _{s \in \mathcal{S}}|y(s)|\right) \sum_{s \in \mathcal{S}} \beta(s)=\sup _{s \in \mathcal{S}}|y(s)|<\infty .
$$

Thus H3 holds. Problem (D3) is similar to $(D)$ in structure, and as in [36], we see it as the (natural) dual of the CILP

$$
\begin{gathered}
(P 3) \inf \sum_{s \in \mathcal{S}} \sum_{a \in A} c(s, a) x(s, a) \\
\sum_{a \in \mathcal{A}} x(s, a)-\alpha \sum_{s^{\prime} \in \mathcal{S}} \sum_{a \in \mathcal{A}} p\left(s \mid s^{\prime}, a\right) x\left(s^{\prime}, a\right)=\beta(s), \text { for } s \in \mathcal{S}, \\
x(s, a) \geq 0, \text { for } s \in \mathcal{S}, a \in \mathcal{A} .
\end{gathered}
$$

An abstract infinite-dimensional LP approach to MDPs is discussed in Chapter 12 of [21]. However, it seems difficult, if not impossible, to directly apply duality results there to countable-state MDPs. Specifically, the abstract results in [21] rely on interior point, or more generally, closedness 

subspaces of $\Re^{\mathbb{N}}[28,31,34]$. To the best of the author's knowledge, papers that apply generic CILP duality results specifically to CILP formulations of infinite-horizon countable-state MDPs under the discounted cost criterion are essentially non-existent. For instance, Ross [36] presented our problems (P3) and (D3) as "duals" without proving any duality results. Puterman [32] did not discuss the LP approach for countable-state MDPs. Evans [11] proved duality results for countable-state MDPs but only for the finite-horizon case. Altman [2] focused on constrained MDPs and presented a counterpart of our problem (P3) for that more general case, but did not explicitly analyze its relation with a counterpart of our problem (D3). Altman [3], in Chapters 8, 9, and 10, focused on constrained MDPs with the total cost and the discounted cost criteria and showed that there is no duality gap between the corresponding primal and the dual LPs (the author thanks Ilbin Lee of the University of Michigan, Ann Arbor, for bringing these Chapters to his attention). Altman's treatment was technically more demanding than and different from our approach here. Lee et al. [26] simplified Altman's approach and applied it to discounted countablestate MDPs with unbounded rewards. The analysis there is entirely different from our presentation here. Specifically, unlike us, Lee et al. do not use weak duality and then complementary slackness to prove strong duality between two explicitly derived complementary solution. Instead, they use the idea of an occupancy measure from Altman, which calls for transforming the discounted expected cost MDP into an expected cost MDP with an absorbing state. They then utilize various known results from Altman, and from Feinberg and Shwartz [21] about this transformed MDP to prove strong duality. As such, the approach in Lee et al. appears more complicated than our proof here. Hordijk and Lasserre [23] and others considered infinite-dimensional LP formulations of countablestate MDPs but they focused on the average cost criterion. Ghate and Smith [15] and Lee et al. [25] derived weak duality, complementary slackness and strong duality for infinite-horizon, finite-state, non-stationary MDPs, which can be seen as a special case of countable-state MDPs. The proofs there exploited the acyclic nature of state transitions, which is a characteristic of non-stationary MDPs (and this does not hold here). In his doctoral dissertation [13], the author proved duality results for CILP formulations of countable-state MDPs under the restrictive assumption that the set of states reachable in one transition from any state-action pair is finite. Finally note that the results in Romeijn et al. [35] and in Romeijn and Smith [34] do not apply here because some of the constraints could include infinitely many variables and some of the variables could appear in infinitely many constraints. We show below that duality results between $(P 3)$ and $(D 3)$ follow easily from our general theory above.

For any feasible variables $x(s, a)$ in (P3), we have, $\sum_{s \in \mathcal{S}} \sum_{a \in \mathcal{A}} x(s, a)=1 /(1-\alpha)$. To see this, by adding the equality constraints in (MDP) over all $s \in \mathcal{S}$, we get,

$$
\sum_{s \in \mathcal{S}} \sum_{a \in \mathcal{A}} x(s, a)-\alpha \sum_{s \in \mathcal{S}} \sum_{s^{\prime} \in \mathcal{S}} \sum_{a \in \mathcal{A}} p\left(s \mid s^{\prime}, a\right) x\left(s^{\prime}, a\right)=\sum_{s \in \mathcal{S}} \beta(s)=1 .
$$

Since $p\left(s \mid s^{\prime}, a\right) x\left(s^{\prime}, a\right) \geq 0$, we can interchange the order of summation in the above equation to get

$$
\sum_{s \in \mathcal{S}} \sum_{a \in \mathcal{A}} x(s, a)-\alpha \sum_{s^{\prime} \in \mathcal{S}} \sum_{a \in \mathcal{A}} \sum_{s \in \mathcal{S}} p\left(s \mid s^{\prime}, a\right) x\left(s^{\prime}, a\right)=1 .
$$

But since $\sum_{s \in \mathcal{S}} p\left(s \mid s^{\prime}, a\right)=1$, the above equation yields $\sum_{s \in \mathcal{S}} \sum_{a \in \mathcal{A}} x(s, a)=1 /(1-\alpha)$ as claimed.

In view of this observation, we choose $X=l_{1}$, the space of absolutely summable sequences, as the variable space for $(P 2)$. For this choice, the series in the objective function converges 
(absolutely) because

$$
\sum_{s \in \mathcal{S}} \sum_{a \in \mathcal{A}}|c(s, a) x(s, a)| \leq c \sum_{s \in \mathcal{S}} \sum_{a \in \mathcal{A}}|x(s, a)|<\infty .
$$

That is, H1 holds. Similarly, for any $x \in l_{1}$, the series $\sum_{s^{\prime} \in \mathcal{S}} \sum_{a \in \mathcal{A}} p\left(s \mid s^{\prime}, a\right) x\left(s^{\prime}, a\right)$ converges (absolutely) because

$$
\sum_{s^{\prime} \in \mathcal{S}} \sum_{a \in \mathcal{A}}\left|p\left(s \mid s^{\prime}, a\right) x\left(s^{\prime}, a\right)\right| \leq \sum_{s^{\prime} \in \mathcal{S}} \sum_{a \in \mathcal{A}}\left|p\left(s \mid s^{\prime}, a\right)\right|\left|x\left(s^{\prime}, a\right)\right| \leq \sum_{s^{\prime} \in \mathcal{S}} \sum_{a \in \mathcal{A}}\left|x\left(s^{\prime}, a\right)\right|<\infty .
$$

Thus, H2 holds.

\subsection{Weak duality and complementary slackness}

We now show that H4, H5 hold for the pair $X=l_{1}$ and $Y=l_{\infty}$. Specifically, consider any $x \in l_{1}$ and any $y \in l_{\infty}$ and a fixed $s \in \mathcal{S}$. We have,

$$
\begin{aligned}
& \sum_{a \in \mathcal{A}}|x(s, a) y(s)|+\sum_{s^{\prime} \in \mathcal{S}} \sum_{a \in \mathcal{A}}\left|-\alpha p\left(s \mid s^{\prime}, a\right) x\left(s^{\prime}, a\right) y(s)\right| \leq \sup _{s \in \mathcal{S}}|y(s)|\left(\sum_{a \in \mathcal{A}}|x(s, a)|+\sum_{s^{\prime} \in \mathcal{S}} \sum_{a \in \mathcal{A}}\left|x\left(s^{\prime}, a\right)\right|\right) \\
& <\infty .
\end{aligned}
$$

Thus, the series $\sum_{a \in \mathcal{A}}|x(s, a) y(s)|+\sum_{s^{\prime} \in \mathcal{S}} \sum_{a \in \mathcal{A}}\left|-\alpha p\left(s \mid s^{\prime}, a\right) x\left(s^{\prime}, a\right) y(s)\right|$ converges to some limit $L_{s}(x, y(s))$ and H4 holds. Moreover, $L_{s}(x, y(s)) \leq \sup _{s \in \mathcal{S}}|y(s)|\left(\sum_{a \in \mathcal{A}}|x(s, a)|+\sum_{s^{\prime} \in \mathcal{S}} \sum_{a \in \mathcal{A}} p\left(s \mid s^{\prime}, a\right)\left|x\left(s^{\prime}, a\right)\right|\right)$. Consequently,

$$
\begin{aligned}
& \sum_{s \in \mathcal{S}} L_{s}(x, y(s)) \leq \sup _{s \in \mathcal{S}}|y(s)|\left(\sum_{s \in \mathcal{S}} \sum_{a \in \mathcal{A}}|x(s, a)|+\sum_{s \in \mathcal{S}} \sum_{s^{\prime} \in \mathcal{S}} \sum_{a \in \mathcal{A}} p\left(s \mid s^{\prime}, a\right)\left|x\left(s^{\prime}, a\right)\right|\right) \\
& =\sup _{s \in \mathcal{S}}|y(s)|(\sum_{s \in \mathcal{S}} \sum_{a \in \mathcal{A}}|x(s, a)|+\sum_{s^{\prime} \in \mathcal{S}} \sum_{a \in \mathcal{A}}\left|x\left(s^{\prime}, a\right)\right| \underbrace{\sum_{s \in \mathcal{S}} p\left(s \mid s^{\prime}, a\right)}_{1}) \\
& =\sup _{s \in \mathcal{S}}|y(s)|\left(\sum_{s \in \mathcal{S}} \sum_{a \in \mathcal{A}}|x(s, a)|+\sum_{s^{\prime} \in \mathcal{S}} \sum_{a \in \mathcal{A}}\left|x\left(s^{\prime}, a\right)\right|\right)<\infty .
\end{aligned}
$$

Thus, $\sum_{s \in \mathcal{S}} L_{s}(x, y(s))$ converges as required for H5. Therefore, Lemma 2.1 applies, and consequently, our weak duality and complementary slackness results in Theorems 2.2 and 2.3 also hold.

\subsection{Strong duality through Bellman's equations}

We now use Bellman's equations to construct a pair of complementary feasible solutions to $(P 3)$ and (D3). The first item in Theorem 2.3 then implies that strong duality holds between $(P 3)$ and $(D 3)$. Suppose $y^{*}(s)$, for $s \in \mathcal{S}$, is an optimal solution to (D3). Recall that such a solution is known to exist from the theory of countable-state MDPs in [36] (in fact, this can also be proven independently from scratch without relying on the relationship between (D3) and the corresponding $\mathrm{MDP})$. As mentioned earlier, $y^{*}(s)$ equals the optimal cost-to-go $v(s)$. It is also known that these optimal costs-to-go uniquely satisfy Bellman's equations

$$
y^{*}(s)=c\left(s, a^{*}(s)\right)+\alpha \sum_{s^{\prime} \in \mathcal{S}} p\left(s^{\prime} \mid s, a^{*}(s)\right) y^{*}\left(s^{\prime}\right), \forall s \in \mathcal{S},
$$



optimal action $a^{*}(s)$ with each state $s \in \mathcal{S}$ to construct an optimal policy $\pi^{*}$. Let $\tilde{A}(s)$ denote the set of actions $a \in \mathcal{A}$ that are not optimal in state $s \in \mathcal{S}$. Then,

$$
y^{*}(s)<c(s, a)+\alpha \sum_{s^{\prime} \in \mathcal{S}} p\left(s^{\prime} \mid s, a\right) y^{*}\left(s^{\prime}\right), \forall a \in \tilde{A}(s), \forall s \in \mathcal{S} .
$$

That is, the inequality constraint in (D3) for state $s$ is not active at $a \in \tilde{A}(s)$ (otherwise this action would be optimal in $s$ ). We define

$$
x^{*}(s, a) \triangleq \sum_{s^{\prime} \in \mathcal{S}} \beta\left(s^{\prime}\right) \sum_{n=1}^{\infty} \alpha^{n-1} P_{\pi^{*}}\left(\mathbf{S}_{n}=s, \mathbf{A}_{n}=a \mid \mathbf{S}_{1}=s^{\prime}\right), \forall s \in \mathcal{S}, \forall a \in \mathcal{A} .
$$

This definition is identical to Equation (6.9.3) in Puterman [32]. Here, we have used the notation $P_{\pi^{*}}\left(\mathbf{S}_{n}=s, \mathbf{A}_{n}=a \mid \mathbf{S}_{1}=s^{\prime}\right)$ to denote the probability that the (random) state $\mathbf{S}_{n}$ at the $n$ decision epoch will be $s$ and the action $\mathbf{A}_{n}$ chosen by policy $\pi^{*}$ in that state will be $a$, given that the (random) initial state $\mathbf{S}_{1}$ was $s^{\prime}$. We make the key observation that $x^{*}(s, a)=0$ for $a \in \tilde{A}(s)$ because $P_{\pi^{*}}\left(\mathbf{S}_{n}=s, \mathbf{A}_{n}=a \mid \mathbf{S}_{1}=s^{\prime}\right)=0$ as policy $\pi^{*}$ does not choose this action in state $s$. This discussion shows that $y^{*}$ and $x^{*}$ are complementary solutions. It remains to prove that $x^{*}$ is feasible to (P3). This in fact follows by replicating the algebra shown in the proof of Theorem 6.9.1(a) on page 225 of Puterman [32] (note that although Puterman's proof is for finite-state MDPs, the steps there are valid in our case as well; this is because all requisite interchanges in the order of sums are allowed by the non-negativity of the summands).

\subsection{A stochastic equipment replacement example}

We conclude this section by applying our duality results on countable-state MDPs to an example adapted from Section 6.10.4 in [32]. Consider a stochastic equipment replacement model where the equipment can be in any one of states $\{0,1,2, \ldots\}$ at the beginning of each time-period. State 0 corresponds to a new equipment; larger states represent poorer equipment conditions. At the beginning of each time-period, the decision-maker can either choose to replace the equipment with a new one (action 0) or keep the existing equipment (action 1). Between two decision epochs, the condition of the equipment worsens by $i \geq 0$ states with probability $q(i)$. This leads to the following transition probabilities:

$$
\begin{aligned}
& p\left(s^{\prime} \mid s, 0\right)=q\left(s^{\prime}\right), s^{\prime} \geq 0, \text { and } \\
& p\left(s^{\prime} \mid s, 1\right)=\left\{\begin{array}{l}
0, \text { if } s^{\prime}<s \\
q\left(s^{\prime}-s\right), \text { if } s^{\prime} \geq s .
\end{array}\right.
\end{aligned}
$$

Observe that these transition probabilities do not satisfy the finite-reachability property assumed in the author's doctoral dissertation [13]. The costs in this model are given by

$$
c(s, 0)=\gamma+h(0), \text { and } c(s, 1)=h(s),
$$

where $\gamma>0$ is the cost of buying a new piece of equipment and $h(s)$ is the cost of operating an equipment in condition $s$ for one period. It is natural to expect that $h(s)$ is non-decreasing in $s$ as it should be cheaper to operate an equipment that is in a better condition. As a special case, we consider the bounded, non-negative, non-decreasing cost function $h(s)=1-\exp (-s)$. Thus, the costs in (57) are bounded between zero and $\gamma+1$. Then, weak duality, complementary slackness, and strong duality hold for CILP formulations of this stochastic equipment replacement example. 


\section{Application to robust auction-design}

CILPs in the existing literature have appeared mostly in the context of infinite-horizon planning problems. In this section, we highlight broader applicability of CILPs and also of our approach to duality through a robust optimization problem.

We first describe the nominal version of this problem. Consider an auctioneer with $I$ units of a product on hand that she wishes to sell in a single, multi-unit Vickrey auction. In this mechanism, the clearing price equals the highest losing bid. Suppose each bidder's bid is independently uniformly distributed over the interval $[0,1]$, and that the number of participating bidders is a random variable $\mathbf{N}$ with support $\{0,1,2, \ldots\}$. We use $x_{j}$ to denote the probability that $\mathbf{N}=j$, for $j=0,1,2, \ldots$. The auctioneer needs to decide the number of units to put up for auction, that is, the auction's lot-size, so as to maximize expected revenue. The key economic trade-off is roughly as follows. For any fixed number of participating bidders, a large lot-size reduces the clearing price for each unit whereas a small lot-size increases the clearing price for each unit; as shown in [9], the resulting expected revenue function is unimodal. Moreover, any remaining units (due to an insufficient number of bidders) can be scrapped at a salvage price of $0 \leq s<1$ per unit and this adds another dimension to the economic trade-off. If the lot-size is $l \in\{0,1,2, \ldots, I\}$, then the expected revenue is given by

$$
\psi(l ; x) \triangleq l \sum_{j=l+1}^{\infty}(1-(l+1) /(j+1)) x_{j}+(I-l) s \sum_{j=l+1}^{\infty} x_{j}+I s \sum_{j=0}^{l} x_{j} .
$$

In this expression, the first term is the expected revenue earned in a "successful" multi-unit Vickrey auction with $l$ units. The term "successful" here means that the number of participating bidders is more than the lot-size. Specifically, in this first term, the expression $(1-(l+1) /(j+1))$ is the expected value of the $l+1$ st largest among $j \geq l+1$ independent uniform $[0,1]$ bids. The second term is the expected revenue earned by selling the remaining units when the auction with $l$ units is successful. The third term equals the expected revenue earned by selling the remaining units when this auction is "unsuccessful". The term "unsuccessful" here means that the number of participating bidders is no more than the lot-size and hence the auction is cancelled, thus returning the $l$ units back to the inventory. A detailed discussion of successful and unsuccessful auctions is included in $[9,30]$. The above formula simplifies to $\psi(l ; x)=l \sum_{j=l+1}^{\infty}((j-l) /(j+1)-s) x_{j}+I s$, and the auctioneer's problem is given by $\max _{l \in\{0,1, \ldots, I\}} \psi(l ; x)$. We refer the reader to [9] for a detailed discussion of this problem and its many extensions to other auction mechanisms and other bid distributions.

Here, we are interested in a robust counterpart of this nominal problem. In this robust counterpart, the auctioneer is uncertain about the probability mass function $x_{j}$, for $j=0,1,2, \ldots$, of N. Following a standard approach in robust optimization [7], we use an interval uncertainty model for these probabilities. Specifically, let $\lambda>0$, and we define a Poisson reference distribution with probability mass function $p_{j}(\lambda)=e^{-\lambda} \lambda^{j} / j$ !, for $j=0,1,2, \ldots$. We assume that the probability mass function belongs to the uncertainty set $\mathcal{X}(\lambda ; \delta)$ given by

$$
\mathcal{X}(\lambda ; \delta) \triangleq\left\{x \geq 0:\left|p_{j}(\lambda)-x_{j}\right| / p_{j}(\lambda) \leq \delta, j=1,2, \ldots ; \sum_{j=0}^{\infty} x_{j}=1\right\},
$$

for some $0<\delta<1$. That is, the probabilities $x_{j}$, for $j=1,2, \ldots$, are within a relative absolute distance $\delta$ from the probabilities $p_{j}(\lambda)$ of the reference Poisson distribution. In our robust counter- 
part, the auctioneer maximizes the worst-case expected revenue over all probability mass functions in $\mathcal{X}(\lambda ; \delta)$. That is, we now wish to solve

$$
\max _{l \in\{0,1, \ldots, I\}} \min _{x \in \mathcal{X}(\lambda ; \delta)} \psi(l ; x)
$$

This robust problem calls for solving a finite set of CILPs. Specifically, for each fixed $l \in\{0,1, \ldots, I\}$, the inner problem $\min _{x \in \mathcal{X}(\lambda ; \delta)} \psi(l ; x)$ is a CILP that we will discuss in detail in this section.

For each fixed lot-size $l \in\{0,1,2, \ldots, I\}$, after ignoring the constant term $I s$ and the multiplier $l$ in the formula for $\psi(l ; x)$, the inner problem is given by

$$
\begin{gathered}
\min \sum_{j=l+1}^{\infty}((j-l) /(j+1)-s) x_{j}, \\
\sum_{j=0}^{\infty} x_{j}=1 \\
(1-\delta) p_{j}(\lambda) \leq x_{j}, j=1,2, \ldots, \\
x_{j} \leq(1+\delta) p_{j}(\lambda), j=1,2, \ldots, \\
x_{j} \geq 0, j=0,1,2, \ldots
\end{gathered}
$$

We first note that when $s=0$, the worst-case distribution is obtained by setting $x_{j}=(1-\delta) p_{j}(\lambda)$ for $j=1,2, \ldots$ and setting $x_{0}=1-(1-\delta)\left(1-e^{-\lambda}\right)$ because the coefficients of $x_{j}$ in the objective function are all non-negative. This is no longer the case when $s>0$.

We let $c_{j}(l) \triangleq 0$, for $j=0,1, \ldots, l$, and $c_{j}(l) \triangleq(j-l) /(j+1)-s$, for $j=l+1, l+2, \ldots$ Note that $0 \leq c_{j}(l) \leq 1$ for all $j$. After subtracting nonnegative surplus variables $u_{j}$ from constraints (60), and adding nonnegative slack variables $v_{j}$ to constraints (61), we obtain the following pair of primal and dual problems $\left(P 4_{l}\right)$ and $\left(D 4_{l}\right)$ that are identical in form to $(P)$ and $(D)$, respectively.

$$
\begin{gathered}
\left(P 4_{l}\right) \min \sum_{j=0}^{\infty} c_{j}(l) x_{j}, \\
\sum_{j=0}^{\infty} x_{j}=1 \\
x_{j}-u_{j}=(1-\delta) p_{j}(\lambda), j=1,2, \ldots, \\
x_{j}+v_{j}=(1+\delta) p_{j}(\lambda), j=1,2, \ldots, \\
x_{j} \geq 0, j=0,1,2, \ldots, \\
u_{j} \geq 0, j=1,2, \ldots, \\
v_{j} \geq 0, j=1,2, \ldots \\
\left(D 4_{l}\right) \max (1-\delta) \sum_{j=1}^{\infty} p_{j}(\lambda) y_{j}+(1+\delta) \sum_{j=1}^{\infty} p_{j}(\lambda) z_{j}+\theta, \\
\theta \leq c_{0}(l), \\
\theta+y_{j}+z_{j} \leq c_{j}(l), j=1,2, \ldots, \\
y_{j} \geq 0, j=1,2, \ldots, \\
z_{j} \leq 0, j=1,2, \ldots
\end{gathered}
$$


Again notice that the results in Romeijn et al. [35] and in Romeijn and Smith [34] do not apply because constraint (64) includes infinitely many variables.

We first choose appropriate sequence spaces for the variables in the above primal-dual pair. Let $X$ be a subspace of $\Re^{\mathbb{N}}$ with the property that for any $x=\left(x_{0}, x_{1}, \ldots\right) \in X$, there exists a real number $\tau(x)$ such that $\left|x_{j}\right| \leq \tau(x) p_{j}(\lambda)$ for $j=1,2, \ldots$. Also let $U$ be a subspace of $\Re^{\mathbb{N}}$ with the property that for any $u=\left(u_{1}, u_{2}, \ldots\right) \in U$, there exists a real number $\tau(u)$ such that $\left|u_{j}\right| \leq \tau(u) p_{j}(\lambda)$ for $j=1,2, \ldots$. We choose the product space $X \times U \times U$ for variables $(x, u, v)$ in $\left(P 4_{l}\right)$. Let $Y$ be a subspace of $\Re^{\mathbb{N}}$ with the property that $\sum_{j=1}^{\infty} p_{j}(\lambda)\left|y_{j}\right|<\infty$ for any $y=\left(y_{1}, y_{2}, \ldots\right) \in Y$. We choose the product space $\Re \times Y \times Y$ for variables $(\theta, y, z)$ in $\left(D 4_{l}\right)$.

For these choices of subspaces for the variables in $\left(P 4_{l}\right)$ and $\left(D 4_{l}\right)$, it is not difficult to confirm that the various series involved converge. For instance, note that, for every $x \in X$,

$$
\sum_{j=0}^{\infty}\left|c_{j}(l) x_{j}\right| \leq \sum_{j=0}^{\infty}\left|c_{j}(l)\right|\left|x_{j}\right| \leq \sum_{j=0}^{\infty}\left|x_{j}\right| \leq\left|x_{0}\right|+\tau(x) \sum_{j=0}^{\infty} p_{j}(\lambda)=\left|x_{0}\right|+\tau(x),
$$

and hence the objective function in $\left(P 4_{l}\right)$ converges (absolutely). Similarly, the series in constraint (64) converges (absolutely). So does the series in the objective function of $\left(D 4_{l}\right)$. In short, H1, H2, H3 hold.

Now consider any fixed $(x, u, v) \in X \times U \times U$ and any fixed $(\theta, y, z) \in \Re \times Y \times Y$. We have,

$$
\sum_{j=0}^{\infty}\left|x_{j}\right||\theta| \leq|\theta|\left|x_{0}\right|+|\theta| \tau(x) \sum_{j=0}^{\infty} p_{j}(\lambda)=|\theta|\left|x_{0}\right|+|\theta| \tau(x) .
$$

Thus, the series $\sum_{j=0}^{\infty}\left|x_{j} \theta\right|$ converges to some limit $L_{\theta}(x)$. Similarly, we let $L_{j}\left(x, u, y_{j}\right)=\left|x_{j} y_{j}\right|+$ $\left|u_{j} y_{j}\right|$, and $L_{j}\left(x, v, z_{j}\right)=\left|x_{j} z_{j}\right|+\left|v_{j} z_{j}\right|$. Thus, H4 holds. Moreover,

$$
\begin{aligned}
& |\theta|\left|x_{0}\right|+|\theta| \tau(x)+\sum_{j=1}^{\infty}\left|x_{j} y_{j}\right|+\sum_{j=1}^{\infty}\left|u_{j} y_{j}\right|+\sum_{j=1}^{\infty}\left|x_{j} z_{j}\right|+\sum_{j=1}^{\infty}\left|v_{j} z_{j}\right| \\
& \leq|\theta| \tau(x)+\tau(x) \sum_{j=1}^{\infty} p_{j}(\lambda)\left|y_{j}\right|+\tau(u) \sum_{j=1}^{\infty} p_{j}(\lambda)\left|y_{j}\right|+\tau(x) \sum_{j=1}^{\infty} p_{j}(\lambda)\left|z_{j}\right|+\tau(v) \sum_{j=1}^{\infty} p_{j}(\lambda)\left|z_{j}\right| \\
& <\infty .
\end{aligned}
$$

As a result, H5 holds and thus Lemma 2.1 applies, and $\left(P 4_{l}\right)$ and $\left(D 4_{l}\right)$ satisfy weak duality and complementary slackness as in Theorems 2.2 and 2.3.

Recall that for the countable-state MDPs above, strong duality was established by explicitly deriving a pair of feasible complementary solutions and then applying the first item from Theorem 2.3. Here we pursue an alternate approach to prove strong duality. We show that conditions C1-C5 in Theorem 3.2 hold for $\left(P 4_{l}\right)$ and $\left(D 4_{l}\right)$.

It is easy to see from the constraints in $\left(P 4_{l}\right)$ that its variables belong to the set

$C(\lambda ; \delta) \triangleq\left\{(x, u, v) \in X \times U \times U: 0 \leq x_{0} \leq 1,(1-\delta) p_{j}(\lambda) \leq x_{j}, u_{j}, v_{j} \leq(1+\delta) p_{j}(\lambda), j=1,2, \ldots\right\}$.

We also have,

Lemma 6.1. There exists a finite constant $M(l)>0$ such that $\theta \geq-M(l)$ without loss of optimality in $\left(D 4_{l}\right)$. Similarly, there exist finite constants $K_{j}(l)>0$ and $L_{j}(l)>0$ such that $y_{j} \leq K_{j}(l)$ and 

belong to the set

$$
\mathcal{K}_{l} \triangleq\left\{(\theta, y, z) \in(\Re \times Y \times Y):|\theta| \leq M(l) ;\left|y_{j}\right| \leq K_{j}(l), j=1,2, \ldots ;\left|z_{j}\right| \leq L_{j}(l), j=1,2, \ldots\right\}
$$

without loss of optimality.

Proof. The objective function in $\left(D 4_{l}\right)$ can be rewritten as $\theta p_{0}(\lambda)+\sum_{j=1}^{\infty} p_{j}(\lambda)\left(y_{j}+z_{j}+\theta\right)+$ $\delta \sum_{j=1}^{\infty} p_{j}(\lambda)\left(z_{j}-y_{j}\right)$. For any feasible solution to $\left(D 4_{l}\right)$, this objective is bounded above by $1+\theta p_{0}(\lambda)$. This follows since $z_{j}-y_{j} \leq 0$ and $y_{j}+z_{j}+\theta \leq c_{j}(l) \leq 1$. Also observe that the solution $y_{j}=z_{j}=0$ for $j=1,2, \ldots$, and $\theta=\inf _{j=0,1, \ldots} c_{j}(l)=\min \{0,(1 /(l+1))-s\}$ is feasible to $\left(D 4_{l}\right)$. Thus, the optimal objective value in $\left(D 4_{l}\right)$ is bounded below by $\min \{0,(1 /(l+1))-s\} \geq-s$. These upper and lower bounds imply that $\theta \geq-(1+s) / p_{0}(\lambda)$ without loss of optimality, as required to establish the first claim.

For the second claim, suppose $y_{1}$ does not have the claimed upper bound. Thus, for each fixed number $K_{1}(l)>0$, there exists an optimal solution with $y_{1}>K_{1}(l)$. Similar to above, we rewrite the objective function as $\theta p_{0}(\lambda)+\sum_{j=1}^{\infty} p_{j}(\lambda)\left(y_{j}+z_{j}+\theta\right)+\delta\left(z_{1}-y_{1}\right) p_{1}(\lambda)+\delta \sum_{j=2}^{\infty} p_{j}(\lambda)\left(z_{j}-y_{j}\right)$. But since $\theta \leq 0, y_{1}+z_{1}+\theta \leq 1,-\theta \leq M(l), z_{1} \leq 0$, and $z_{j}-y_{j} \leq 0$, the objective function is bounded above by $1-\delta p_{1}(\lambda) y_{1}$. Then, by choosing a large enough $y_{1}$, we can make the optimal objective value smaller than $-2 s$. This contradicts the fact that the optimal objective value is at least $-s$.

For the third claim, suppose $z_{1}$ does not have the claimed upper bound. Thus, for each fixed number $L_{1}(l)>0$, there exists an optimal solution with $z_{1}<-L_{1}(l)$. Again, by algebra similar to above, the objective function value can be bounded above by $1+\delta p_{1}(\lambda) z_{1}$. By choosing a sufficiently negative $z_{1}$, we can make the optimal objective value smaller than $-2 s$ to get a contradiction.

We use $\left(P 4_{l}(n)\right)$ to denote the finite-dimensional truncation of $\left(P 4_{l}\right)$ that only includes nonnegative variables $x_{0}, x_{1}, \ldots, x_{n} ; u_{1}, u_{2}, \ldots, u_{n} ; v_{1}, v_{2}, \ldots, v_{n}$; constraint (64); and constraints (65) and (66) for $j=1,2, \ldots, n$. We use $\left(D 4_{l}(n)\right)$ to denote its dual. It is easy to see that both these problems have feasible solutions and the variable bounds in sets $\mathcal{C}_{l}$ and $\mathcal{K}_{l}$ also hold for $\left(P 4_{l}(n)\right)$ and $\left(D 4_{l}(n)\right)$. Thus, these problems have optimal solutions that belong to sets $\mathcal{C}_{l}$ and $\mathcal{K}_{l}$. Moreover, $\mathcal{C}_{l}$ and $\mathcal{K}_{l}$ are compact. This shows that conditions $\mathrm{C} 1, \mathrm{C} 2$, and $\mathrm{C} 5$ hold. Condition $\mathrm{C} 4$ holds because each constraint in $\left(D 4_{l}\right)$ includes at most three variables. To show that condition C3 holds, we prove that the series $\sum_{j=0}^{\infty} x_{j}$ converges uniformly over $\mathcal{C}_{l}$. To see this, first note that for any $(x, u, v) \in \mathcal{C}_{l}$, we have, $\left|x_{0}\right| \leq 1$, and $\left|x_{j}\right| \leq(1+\delta) p_{j}(\lambda)$, for $j=1,2, \ldots$ As a result, $\sum_{j=0}^{\infty}\left|x_{j}\right| \leq 1+(1+\delta) \sum_{j=1}^{\infty} p_{j}(\lambda) \leq 3$. Uniform convergence then follows from the Weierstrass test.

Our nominal problem here focused on choosing the lot-size in a single auction. An extension of this nominal problem to sequential auctions was studied in [9]. This extension used a stochastic dynamic programming formulation. The robust counterpart of this lot-size selection problem in sequential auctions could also be developed by applying the theory of robust dynamic programming. The inner problem in the resulting robust Bellman's equations will also be a CILP, and our main ideas in this section will be applicable to that inner problem as well.

Finally, although our results in Sections 2 and 3 were illustrated here via a robust auction-design problem, these ideas will also apply to inner problems in other more general robust optimization problems that employ the interval uncertainty model using a probability mass function with countable support. 


\section{Acknowledgments}

Funded in part by the National Science Foundation through grant \#CMMI 1333260. The author is indebted to Professor Robert L Smith of the University of Michigan, Ann Arbor, USA, for generously providing his insights into infinite-dimensional optimization through numerous discussions over the last several years.

\section{References}

[1] C D Aliprantis and K C Border. Infinite-dimensional analysis: a hitchhiker's guide. SpringerVerlag, Berlin, Germany, 1994.

[2] E Altman. Denumerable constrained markov decision processes and finite approximations. Mathematics of Operations Research, 19(1):169-191, 1994.

[3] E Altman. Constrained Markov decision processes. Stochastic Modeling. Chapman and Hall/CRC, Boca Raton, FL, USA, 1999.

[4] E J Anderson and P Nash. Linear programming in infinite-dimensional spaces: theory and applications. John Wiley and Sons, Chichester, UK, 1987.

[5] T M Apostol. Mathematical Analysis. Addison Wesley, Reading, MA, USA, 1974.

[6] A Basu, K Martin, and C T Ryan. On the sufficiency of finite support duals in semi-infinite linear programming. Operations Research Letters, 42(1):16-20, 2014.

[7] A Ben-Tal, L El Ghaoui, and A Nemirovski. Robust Optimization. Princeton University Press, Princeton, New Jersey, USA, 2009.

[8] D Bertsimas and J N Tsitsiklis. Introduction to linear optimization. Athena Scientific, Belmon, MA, USA, 1997.

[9] X Chen, A Ghate, and A K Tripathi. Dynamic lot-sizing in sequential online retail auctions. European Journal of Operational Research, 215(1):257-267, 2011.

[10] G Dantzig. Linear programming and extensions. Princeton University Press, Princeton, New Jersey, USA, 1963.

[11] J P Evans. Duality in Markov decision problems with countable action and state spaces. Management Science, 15(11):626-638, 1969.

[12] D Gale. Theory of linear economic models. University of Chicago Press, Chicago, IL, USA, 1989.

[13] A Ghate. Markov chains, game theory, and infinite programming: three paradigms for optimization of complex systems. PhD thesis, University of Michigan, Ann Arbor, MI, USA, 2006.

[14] A Ghate, D Sharma, and R L Smith. A shadow simplex method for infinite linear programs, forthcoming. Operations Research, 58(4):865-877, 2010.

[15] A Ghate and R L Smith. A linear programming approach to nonstationary infinite horizon markov decision processes. Operations Research, 61(2):413-425, 2013. 
[16] R C Grinold. Infinite horizon programs. Management Science, 18(3):157-170, 1971.

[17] R C Grinold. Finite horizon approximations of infinite horizon linear programs. Mathematical Programming, 12(1):1-17, 1977.

[18] R C Grinold. Convex infinite horizon programs. Mathematical Programming, 25(1):64-82, 1983.

[19] R C Grinold and D S P Hopkins. Computing optimal solutions for infinite-horizon mathematical programs with a transient stage. Operations Research, 21(1):179-187, 1973.

[20] R C Grinold and D S P Hopkins. Duality overlap in infinite linear programs. Journal of Mathematical Analysis and Applications, 41:333-335, 1973.

[21] O Hernandez-Lerma and J B Lasserre. The linear programming approach. In E Feinberg and A Shwartz, editors, Handbook of Markov decision processes: methods and algorithms, chapter 12, pages 377-408. Kluwer, Boston, MA, USA, 2002.

[22] D S P Hopkins. Sufficient conditions for optimality in infinite horizon linear economic models. Technical report, Operations Research House, Stanford University, CA, USA, 1969.

[23] A Hordijk and J B Lasserre. Linear programming formulation of MDPs in countable state space: the multichain case. Mathematical Methods of Operations Research, 40(1):91-108, 1994.

[24] P Jones, J Zydiak, and W Hopp. Stationary dual prices and depreciation. Mathematical Programming, 41(1-3):357-366, 1988.

[25] I Lee, M A Epelman, H E Romeijn, and R L Smith. A linear programming approach to constrained nonstationary infinite-horizon Markov decision processes. Technical Report TR1301, University of Michigan, Ann Arbor, MI, USA, 2013.

[26] I Lee, M A Epelman, H E Romeijn, and R L Smith. A linear programming approach to countable-state discounted markov decision processes with unbounded rewards. http://www . optimization-online.org/DB_HTML/2014/11/4645.html, 2014.

[27] D G Luenberger. Optimization by vector space methods. John Wiley and Sons, New York, NY, USA, 1969.

[28] K Martin, C T Ryan, and M Stern. The Slater Conundrum: Duality and Pricing in Infinite Dimensional Optimization. http://faculty.chicagobooth.edu/kipp.martin/root/Slater Conundrum.pdf, April 2014.

[29] K G Murty. Linear Programming. Wiley, New York, NY, USA, 1983.

[30] E Pinker, A Seidmann, and Y Vakrat. Managing online auctions: Current business and research issues. Management Science, 49(11):1457-1484, 2003.

[31] J Ponstein. On the use of purely finitely additive multipliers in mathematical programming. Journal of Optimization Theory and Applications, 33(1):37-55, 1981.

[32] M L Puterman. Markov decision processes : Discrete stochastic dynamic programming. John Wiley and Sons, New York, NY, USA, 1994. 
[33] H E Romeijn, D Sharma, and R L Smith. Extreme point solutions for infinite network flow problems. Networks, 48(4):209-222, 2006.

[34] H E Romeijn and R L Smith. Shadow prices in infinite dimensional linear programming. Mathematics of Operations Research, 23(1):239-256, 1998.

[35] H E Romeijn, R L Smith, and J C Bean. Duality in infinite dimensional linear programming. Mathematical Programming, 53(1-3):79-97, 1992.

[36] S M Ross. Introduction to stochastic dynamic programming. Academic Press, New York, NY, USA, 1983.

[37] I E Schochetman and R L Smith. Infinite horizon optimization. Mathematics of Operations Research, 14(3):559-574, 1989.

[38] T C Sharkey and H E Romeijn. A simplex algorithm for minimum cost network flow problems in infinite networks. Networks, 52(1):14-31, 2008.

[39] A E Taylor and D C Lay. Introduction to functional analysis. Robert E Krieger Publishing Company, Malabar, FL, USA, 1986. 